\title{
The Priority Heuristic: Making Choices Without Trade-Offs
}

\author{
Eduard Brandstätter \\ Johannes Kepler University of Linz
}

\author{
Gerd Gigerenzer \\ Max Planck Institute for Human Development
}

\author{
Ralph Hertwig \\ University of Basel
}

\begin{abstract}
Bernoulli's framework of expected utility serves as a model for various psychological processes, including motivation, moral sense, attitudes, and decision making. To account for evidence at variance with expected utility, the authors generalize the framework of fast and frugal heuristics from inferences to preferences. The priority heuristic predicts (a) the Allais paradox, (b) risk aversion for gains if probabilities are high, (c) risk seeking for gains if probabilities are low (e.g., lottery tickets), (d) risk aversion for losses if probabilities are low (e.g., buying insurance), (e) risk seeking for losses if probabilities are high, (f) the certainty effect, (g) the possibility effect, and (h) intransitivities. The authors test how accurately the heuristic predicts people's choices, compared with previously proposed heuristics and 3 modifications of expected utility theory: security-potential/aspiration theory, transfer-of-attentionexchange model, and cumulative prospect theory.
\end{abstract}

Keywords: risky choice, heuristics, decision making, frugality, choice process

Conventional wisdom tells us that making decisions becomes difficult whenever multiple priorities, appetites, goals, values, or simply the attributes of the alternative options are in conflict. Should one undergo a medical treatment that has some chance of curing a life-threatening illness but comes with the risk of debilitating side effects? Should one report a crime committed by a friend? Should one buy an expensive, high-quality camera or an inexpensive, low-quality camera? How do people resolve conflicts, ranging from the prosaic to the profound?

The common denominator of many theories of human behavior is the premise that conflicts are mastered by making trade-offs. Since the Enlightenment, it has been believed that weighting and summing are the processes by which such trade-offs can be made in a rational way. Numerous theories of human behavior-including expected value theory, expected utility theory, prospect theory,

Eduard Brandstätter, Department of Psychology, Johannes Kepler University of Linz, Linz, Austria; Gerd Gigerenzer, Center for Adaptive Behavior and Cognition, Max Planck Institute for Human Development, Berlin, Germany; Ralph Hertwig, Faculty of Psychology, University of Basel, Basel, Switzerland.

Ralph Hertwig was supported by Swiss National Science Foundation Grant 100013-107741/1. We thank Will Bennis, Michael Birnbaum, Jerome Busemeyer, Uwe Czienskowski, Ido Erev, Claudia González Vallejo, Robin Hogarth, Eric Johnson, Joseph Johnson, Konstantinos Katsikopoulos, Anton Kühberger, Lola Lopes, Robin Pope, Drazen Prelec, and Lael Schooler for many helpful comments and fruitful discussions, and Uwe Czienskowski a second time for checking the statistical analyses. We are also grateful to Barbara Mellers for providing us with the opportunity to analyze her data and to Florian Sickinger for his help in running the response time experiment.

Correspondence concerning this article should be addressed to Eduard Brandstätter, Department of Psychology, Johannes Kepler University of Linz, Altenbergerstr. 69, 4040, Linz, Austria. E-mail: eduard.brandstaetter@jku.at
Benjamin Franklin's moral algebra, theories of moral sense such as utilitarianism and consequentionalism (Gigerenzer, 2004), theories of risk taking (e.g., Wigfield \& Eccles, 1992), motivational theories of achievement (Atkinson, 1957) and work behavior (e.g., Vroom, 1964), theories of social learning (Rotter, 1954), theories of attitude formation (e.g., Fishbein \& Ajzen, 1975), and theories of health behavior (e.g., Becker, 1974; for a review see Heckhausen, 1991) — rest on these two processes. Take how expected utility theory would account for the choice between two investment plans as an example. The reasons for choosing are often negatively correlated with one another. High returns go with low probabilities, and low returns go with high probabilities. According to a common argument, negative correlations between reasons cause people to experience conflict, leading them to make tradeoffs (Shanteau \& Thomas, 2000). In terms of expected utility, the trade-off between investment plans is performed by weighting the utility of the respective monetary outcomes by their probabilities and by summing across the weighted outcomes of each plan. The plan chosen is that with the higher expected utility.

Weighting and summing are processes that have been used to define not only rational choice but also rational inference (Gigerenzer \& Kurz, 2001). In research on inference, weighting was the first to be challenged. In the 1970s and 1980s, evidence emerged that simple unit weights such as +1 and -1 often yield the same predictive accuracy - that is, the same ability to predict rather than simply "postdict," or fit-as the "optimal" weights in multiple regression (Dawes, 1979). According to these results, weighting does not seem to affect predictive accuracy as long as the weight has the right sign.

Next, summing was called into question. The 1990s brought evidence that the predictive accuracy of lexicographic heuristics can be as high as or higher than the accuracy of complex strategies that perform both weighting and summing. This was shown for 
both inferences (e.g., Gigerenzer \& Goldstein, 1996; Gigerenzer, Todd, \& the ABC Research Group, 1999) and preferences (e.g., Payne, Bettman, \& Johnson, 1993). The heuristics in question order attributes-which can be seen as a simple form of weighting - but do not sum them. Instead, they rely on the first attribute that allows for a decision. These results suggest that summing is not always necessary for good reasoning. In addition, some of the environmental structures under which weighting (ordering) without summing is ecologically rational have been identified (Hogarth \& Karelaia, 2005; Katsikopoulos \& Martignon, in press; Martignon \& Hoffrage, 2002; Payne et al., 1993).

Here is the question that concerns us: If, as the work just reviewed demonstrates, both summing without weighting and weighting without summing can be as accurate as weighting and summing, why should humans not use these simpler heuristics? Specifically, might human choice that systematically contradicts expected utility theory be a direct consequence of people's use of heuristics? The success of a long tradition of theories seems to speak against this possibility. Although deviations between the theory of expected utility and human behavior have long since been experimentally demonstrated, psychologists and economists have nevertheless retained the weighting and summing core of the theory, but they have adjusted the functions to create more complex models such as prospect theory and security-potential/aspiration theory. In this article, we demonstrate that a simple heuristic that forgoes summing and therefore does not make trade-offs can account for choices that are anomalies from the point of view of expected utility theory. In fact, it does so in the very gambling environments that were designed to demonstrate the empirical validity of theories of risky choice that assume both weighting and summing. By extension, we suggest that other areas of human decision making that involve conflicting goals, values, appetites, and motives may likewise be explicable in terms of simple heuristics that forgo complex trade-offs.

\section{The Bernoulli Framework and Its Modifications}

Very few great ideas have an exact date of origin, but the theory of mathematical probability does. In the summer of 1654, the French mathematicians Blaise Pascal and Pierre Fermat exchanged letters on gambling problems posed by a notorious gambler and man-about-town, the Chevalier de Méré. This exchange resulted in the concept of mathematical expectation, which at the time was believed to capture the nature of rational choice (Hacking, 1975). In modern notation, the principle of choosing the option with the highest expected value $(E V)$ is defined as

$$
E V=\sum p_{\mathrm{i}} x_{\mathrm{i}},
$$

where $p_{\mathrm{i}}$ and $x_{\mathrm{i}}$ are the probability and the amount of money, respectively, of each outcome $(i=1, \ldots, n)$ of a gamble. The expected value theory was a psychological theory of human reasoning, believed to describe the reasoning of the educated homme éclairé.

Despite its originality and elegance, the definition of a rational decision by EV soon ran into trouble when Nicholas Bernoulli, a professor of law in Basel, posed the perplexing St. Petersburg paradox. To solve the paradox, his cousin Daniel Bernoulli (1738/ 1954) retained the core of the expected value theory but suggested replacing objective money amounts with subjective utilities. In his view, the pleasure or utility of money did not increase linearly with the monetary amount; instead, the increases in utility declined. This phenomenon entered psychophysics a century later in the form of the Weber-Fechner function (Gigerenzer \& Murray, 1987), and it entered economics in the form of the concept of diminishing returns (Menger, 1871/1990). Daniel Bernoulli modeled the relation between objective and subjective value of money in terms of a logarithmic function. In modern terminology, the resulting expected utility $(E U)$ is defined as

$$
E U=\sum p_{\mathrm{i}} u\left(x_{\mathrm{i}}\right),
$$

where $u\left(x_{\mathrm{i}}\right)$ is a monotonically increasing function defined on objective money amounts $x_{\mathrm{i}}$. At the time of Daniel Bernoulli, the maximization of expected utility was considered both a description and prescription of human reasoning. The present-day distinction between these two concepts, which seems so obvious to researchers today, was not made, because the theory was identical with its application, human reasoning (Daston, 1988). However, the "rational man" of the Enlightenment was dismantled around 1840, when probability theory ceased to be generally considered a model of human reasoning (Gigerenzer et al., 1989). One motive for the divorce between expected utility and human reasoning was apparent human irrationality, especially in the aftermath of the French Revolution. Following the demise of expected utility, psychological theories of thinking virtually ignored the concept of expected utility as well as the laws of probability until the 1950s. The revival of expected utility began with von Neumann and Morgenstern (1947), who based expected utility on axioms. After their landmark book appeared, followed by influential publications such as Edwards (1954, 1962) and Savage (1954) on subjective expected utility, theories of the mind once again started to model human reasoning and choice in terms of probabilities and the expected utility framework (e.g., Fishbein \& Ajzen, 1975; Heckhausen, 1991).

However, it was not long until the first experiments were conducted to test whether people's choices actually follow the predictions of expected utility. Evidence emerged that people systematically violated expected utility theory (Allais, 1953; Ellsberg, 1961; MacCrimmon, 1968; Mosteller \& Nogee, 1951; Preston \& Baratta, 1948), and this evidence has accumulated in the subsequent decades (see Camerer, 1995; Edwards, 1968; Kahneman \& Tversky, 2000). Although specific violations of expected utility, including their normative status, are still under debate (Allais, 1979; Hogarth \& Reder, 1986), there is widespread consensus among experimental researchers that not all of the violations can be explained away.

This article is concerned with how to react to these empirical demonstrations that human behavior often contradicts expected utility theory. So far, two major reactions have surfaced. The first is to retain expected utility theory, by arguing that the contradictory evidence will not generalize from the laboratory to the real world. The arguments for this assertion include that in most of the experiments, participants were not paid contingent on their performance (see Hertwig \& Ortmann, 2001) or were not paid enough to motivate them to behave in accordance with expected utility and that outside the laboratory, market pressures will largely eliminate behavior that violates expected utility theory (see Hogarth \& Reder, 1986). This position is often reinforced by the argument 
that even if one accepts the empirical demonstrations, no powerful theoretical alternative to expected utility exists, and given that all theories are false idealizations, a false theory is still better than no theory.

The second reaction has been to take the data seriously and, just as Bernoulli did, to modify the theory while retaining the original expected utility scaffolding. Examples include disappointment theory (Bell, 1985; Loomes \& Sugden, 1986), regret theory (Bell, 1982; Loomes \& Sugden, 1982), the transfer-of-attentionexchange model (Birnbaum \& Chavez, 1997), decision affect theory (Mellers, 2000), prospect theory (Kahneman \& Tversky, 1979), and cumulative prospect theory (Tversky \& Kahneman, 1992). These theories are noteworthy attempts to adjust Bernoulli's framework to the new empirical challenges by adding one or more adjustable parameters. They represent a "repair" program that introduces psychological variables such as emotions and reference points to rescue the Bernoullian framework (Selten, 2001).

Despite their differences, all of these modifications retain the assumption that human choice can or should be modeled in the same terms that Bernoulli used: that people behave as if they multiplied some function of probability and value, and then maximized. Because of the complex computations involved in some of these modifications, they have often been interpreted to be as-if models. That is, they describe and ideally predict choice outcomes but do not explain the underlying process. The originators of prospect theory, for instance, set themselves the goal "to assemble the minimal set of modifications of expected utility theory that would provide a descriptive account of . . . choices between simple monetary gambles" (Kahneman, 2000, p. x). Prospect theory deals with empirical violations of expected utility by introducing new functions that require new adjustable parameters. For instance, a nonlinear function $\pi$ was added to transform objective probabilities (assuming "regular prospects"):

$$
V=\sum \pi\left(p_{\mathrm{i}}\right) v\left(x_{\mathrm{i}}\right)
$$

where $V$ represents the value of a prospect. The decision weights $\pi\left(p_{\mathrm{i}}\right)$ are obtained from the objective probabilities by a nonlinear, inverse S-shaped weighting function. Specifically, the weighting function $\pi$ overweights small probabilities and underweights moderate and large ones (resulting in an inverse $S$ shape). The value function $v\left(x_{\mathrm{i}}\right)$ is an $\mathrm{S}$-shaped utility function. Just as Bernoulli introduced individual psychological factors (diminishing returns and a person's wealth) to save the expected value framework, Kahneman and Tversky (1979) postulated $\pi$ and $v$ to account for the old and new discrepancies. In the face of new empirical discrepancies and to extend prospect theory to gambles with more than three outcomes, Tversky and Kahneman (1992) further modified prospect theory into cumulative prospect theory.

The essential point is that the weighting function (defined by two adjustable parameters in cumulative prospect theory) and the value function (defined by three adjustable parameters) interpret people's choices that deviate from Bernoulli's framework within that very same framework. For example, the empirical shape of the weighting function is inferred by assuming a multiplication calculus. Overweighting small probabilities, for instance, is an interpretation of people's cognition within Bernoulli's framework-it is not the empirical phenomenon itself. The actual phenomenon is a systematic pattern of choices, which can be accounted for without reference to functions that overweight or underweight objective probabilities. We demonstrate this in the alternative framework of heuristics. The aim of models of heuristics is to both describe the psychological process and predict the final choice.

\section{Heuristics in Risky Choice}

In this article, we pursue a third way to react to the discrepancy between empirical data and expected utility theory: to explain choice as the direct consequence of the use of a heuristic. Unlike proponents of expected utility who dismiss the empirical data (e.g., de Finetti, 1979), we take the data seriously. In fact, we test whether a sequential heuristic can predict classic violations of expected utility as well as four major bodies of choice data. Heuristics model both the choice outcome and the process, and there is substantial empirical evidence that people's cognitive processes and inferences can be predicted by models of heuristics (e.g., Bröder, 2000; Bröder, 2003; Bröder \& Schiffer, 2003; Dhami, 2003; Huber, 1982; Newell, Weston, \& Shanks, 2003; Payne et al., 1993; Payne, Bettman, \& Luce, 1996; Rieskamp \& Hoffrage, 1999; Schkade \& Johnson, 1989).

\section{Which Heuristic?}

Two classes of heuristics are obvious candidates for twoalternative choice problems: lexicographic rules and tallying (Gigerenzer, 2004). Lexicographic rules order reasons-probabilities and outcomes-according to some criterion, search through $m \geq 1$ reasons, and ultimately base the decision on one reason only. The second class, tallying, assigns all reasons equal weights, searches through $m \geq 2$ reasons, and chooses the alternative that is supported by most reasons. For choices between gambles, the empirical evidence suggests that people do not treat the reasons equally, which speaks against the tallying family of heuristics (Brandstätter \& Kühberger, 2005; Deane, 1969; Loewenstein, Weber, Hsee, \& Welch, 2001; Sunstein, 2003). This result was confirmed in the empirical tests reported below. We are then left with a heuristic from the class of lexicographic rules and two questions. First, what are the reasons and in what order are they examined? Second, when is examination stopped? Based on the empirical evidence available, our first task is to derive a candidate heuristic from the set of all possible heuristics.

\section{Priority Rule: In What Order Are Reasons Examined?}

First we consider simple monetary gambles of the type "a probability $p$ to win amount $x$; a probability $(1-p)$ to win amount $y$ " $(x, p ; y)$. Here, the decision maker is given four reasons: the maximum gain, the minimum gain, and their respective probabilities (for losses, see below). All reasons are displayed simultaneously; they are available at no cost. Thus, unlike in tasks for which information needs to be searched in memory (Gigerenzer \& Goldstein, 1996) or in the environment (such as search in external information stores), all the relevant information is fully displayed in front of the participant. The resulting choices are thus "decisions from description" and not "decisions from experience" (Hertwig, Barron, Weber, \& Erev, 2004). The priority rule refers to the order in which people go through the reasons after screening all of them once to make their decision. 
Four reasons result in 24 possible orderings. Fortunately, there are logical and empirical constraints. First, in two-outcome gambles, the two probabilities are complementary, which reduces the number of reasons to three. This in turn reduces the number of possible orders from 24 to 6 . The number can be further constrained by empirical evidence. What is perceived as more important, outcomes or probabilities?

The primacy of outcome over probability had already been noted in Arnauld and Nicole's (1662/1996) Enlightenment classic on the art of thinking. As an example, lottery buyers tend to focus on big gains rather than their tiny probabilities, which is historically grounded in the fact that winning the lottery was one of the very few ways to move upward socially in traditional European societies (Daston, 1988). Similarly, empirical research indicates that emotional outcomes tend to override the impact of probabilities (Sunstein, 2003). Loewenstein et al. (2001) suggest that, in the extreme, people neglect probabilities altogether and instead base their choices on the immediate feelings elicited by the gravity or benefit of future events. Similarly, Deane (1969) reported that anxiety (as measured by cardiac activity) concerning a future electric shock was largely influenced by the intensity of the shock, not by the probability of its occurrence. A series of choice experiments supports the hypothesis that outcome matters more than probability (Brandstätter \& Kühberger, 2005). ${ }^{1}$

From these studies, we assume that the first reason for choosing is one of the two outcomes, not the probability. This reduces the number of orders once again, from six to four. But which outcome is considered first, the minimum or the maximum outcome? The empirical evidence seems to favor the minimum outcome. The frequent observation that people tend to be risk averse in the gain domain (Edwards, 1954) is consistent with ranking the minimum outcome first. This is because the reason for focusing on the minimum outcome is to avoid the worst outcome. In contrast, ranking the maximum outcome first would imply that people are risk seeking with gains - an assumption for which little empirical evidence exists. Further empirical support is given by research documenting that people try to avoid disappointment (from ending up with the worst possible outcome of the chosen gamble) and regret (from obtaining an inferior outcome compared with the alternative not chosen). This motivation to avoid winning nothing (or the minimum amount) is incorporated in regret theory (Loomes \& Sugden, 1982), disappointment theory (Bell, 1985), and in the motivation for avoidance of failure (Heckhausen, 1991).

We conclude that the empirical evidence favors the minimum gain. This reduces the number of possible orders of reasons from four to two. To distinguish between the two remaining orders, we conducted an experiment in which the minimal outcome was held constant, and thus all decisions depended on maximum gains and the probabilities of the minimum gains. These two reasons always suggested opposite choices. Forty-one students from the University of Linz, Austria (22 women, 19 men; $M=23.2$ years, $S D=$ 5.3 years) were tested on four problems:

$(500, .50)$ and $(2,500, .10)[88 \%]$

$(220, .90)$ and $(500, .40)[80 \%]$

$(5,000, .50)$ and $(25,000, .10)[73 \%]$

$(2,200, .90)$ and $(5,000, .40)[83 \%]$
For instance, the first choice was between $€ 500$ (US\$600) with $p=$ .50 , otherwise nothing, and $€ 2,500$ (US $\$ 3,000$ ) with $p=.10$, otherwise nothing. Faced with this choice, 36 of 41 participants $(88 \%)$ selected this first gamble, which has the smaller probability of the minimum gain but the lower maximum gain. On average, $81 \%$ of the participants chose the gamble with the smaller probability of the minimum gain. This result suggests the probability of the minimum gain - rather than the maximum gain —as the second reason. The same conclusion is also suggested by another study in which the experimenters held the minimum outcomes constant across gambles (Slovic, Griffin, \& Tversky, 1990; Study 5). Thus, in the priority rule, below, we propose the following order in which the reasons are attended to:

Priority Rule. Consider reasons in the order: minimum gain, probability of minimum gain, maximum gain.

\section{Stopping Rule: What Is a Good-Enough Reason?}

Heuristic examination is limited rather than exhaustive. Limited examination makes heuristics different from expected utility theory and its modifications, which have no stopping rules and integrate all pieces of information in the final choice. A stopping rule defines whether examination stops after the first, second, or third reason. Again, we consult the empirical evidence to generate a hypothesis about the stopping rule.

What difference in minimum gains is good enough ("satisficing") to stop examination and decide between the two gambles solely on the basis of this information? Just as in Simon's (1983) theory of satisficing, in which people stop when an alternative surpasses an aspiration level (see also Luce, 1956), our use of the term aspiration level refers to the amount that, if met or exceeded, stops examination of reasons. Empirical evidence suggests that the aspiration level is not fixed but increases with the maximum gain (Albers, 2001). For instance, consider a choice between winning $\$ 200$ with probability .50 , otherwise nothing $(\$ 200, .50)$, and winning $\$ 100$ for sure $(\$ 100)$. The minimum gains are $\$ 0$ and $\$ 100$, respectively. Now consider the choice between $\$ 2,000$ with probability $.50(\$ 2,000, .50)$ and $\$ 100$ for sure $(\$ 100)$. The minimum gains still differ by the same amount, the probabilities are the same, but the maximum outcomes differ. People who select the sure gain in the first pair may not select it in the second. Thus, the difference between the minimum gains that is considered large enough to stop examination after the first reason should be dependent on the maximum gain.

A simple way to incorporate this dependency is to assume that people intuitively define it by their cultural number system, which is the base-10 system in the Western world (Albers, 2001). This leads to the following hypothesis for the stopping rule:

\footnotetext{
${ }^{1}$ The results depend on the specific set of gambles: When one of the reasons is not varied, it is not likely that people attend to this reason. For instance, in a "dublex gamble" (Payne \& Braunstein, 1971; Slovic \& Lichtenstein, 1968), one can win $\$ x$ with probability $p_{1}$ (otherwise nothing), and lose $\$ y$ with probability $p_{2}$ (otherwise nothing). Here, the minimum gain of the winning gamble and the minimum loss of the losing gamble are always zero, rendering the minimum outcomes uninformative. Similarly, Slovic et al. (1990) argued that probabilities were more important than outcomes, but here again all minimum outcomes were zero.
} 
Stopping Rule. Stop examination if the minimum gains differ by $1 / 10$ (or more) of the maximum gain.

The hypothesis is that $1 / 10$ of the maximum gain, that is, one order of magnitude, is "good enough." Admittedly, this value of the aspiration level is a first, crude estimate, albeit empirically informed. The aspiration level is a fixed (not free) parameter. If there is an independent measure of individual aspiration levels in further research, the estimate can be updated, but in the absence of such an independent measure, we do not want to introduce a free parameter. We refer to this value as the aspiration level. For illustration, consider again the choice between winning $\$ 200$ with probability .50, otherwise nothing $(\$ 200, .50)$, and winning $\$ 100$ for sure $(\$ 100)$. Here, $\$ 20$ is "good enough." The difference between the minimum gains exceeds this value $(\$ 100>\$ 20)$, and therefore examination is stopped. Information concerning probabilities is not used for the choice.

What if the maximum amount is not as simple as 200 but is a number such as 190? Extensive empirical evidence suggests that people's numerical judgments are not fine-grained but follow prominent numbers, as summarized in Albers (2001). Prominent numbers are defined as powers of 10 (e.g., 1, 10, 100, ..), including their halves and doubles. Hence, the numbers 1, 2, 5, 10, 20, 50, 100, 200, and so on, are examples of prominent numbers. They approximate the Weber-Fechner function in a culturally defined system. We assume that people scale the maximum gain down by $1 / 10$ and round this value to the closest prominent number. Thus, if the maximum gain were $\$ 190$ rather than $\$ 200$, the aspiration level would once again be $\$ 20$ (because $\$ 19$ is rounded to the next prominent number).

If the difference between minimum gains falls short of the aspiration level, the next reason is examined. Again, examination is stopped if the two probabilities of the minimum gains differ by a "large enough" amount. Probabilities, unlike gains, have upper limits and hence are not subject to the Weber-Fechner property of decreasing returns (Banks \& Coleman, 1981). Therefore, unlike for gains, the aspiration level need not be defined relative to the maximum value. We define the aspiration level as $1 / 10$ of the probability scale, that is, one order of magnitude: The probabilities need to differ by at least 10 percentage points to stop examination. This leads to the following hypothesis for the stopping rule:

Stopping Rule. Stop examination if probabilities differ by $1 / 10$ (or more) of the probability scale.

If the differences in the minimum outcomes and their probabilities do not stop examination, then finally the maximum outcomewhichever is higher-decides. No aspiration level is needed.

\section{The Priority Heuristic}

The priority and stopping rules combine to the following process model for two-outcome gambles with nonnegative prospects (all outcomes are positive or zero). We refer to this process as the priority heuristic because it is motivated by first priorities, such as to avoid ending up with the worst of the two minimum outcomes. The heuristic consists of the following steps:

Priority Rule. Go through reasons in the order: minimum gain, probability of minimum gain, maximum gain.
Stopping Rule. Stop examination if the minimum gains differ by $1 / 10$ (or more) of the maximum gain; otherwise, stop examination if probabilities differ by $1 / 10$ (or more) of the probability scale.

Decision Rule. Choose the gamble with the more attractive gain (probability).

The term attractive refers to the gamble with the higher (minimum or maximum) gain and the lower probability of the minimum gain The priority heuristic models difficult decisions, not all decisions It does not apply to pairs of gambles in which one gamble dominates the other one, and it also does not apply to "easy" problems in which the expected values are strikingly different (see the General Discussion section).

The heuristic combines features from three different sources: Its initial focus is on outcomes rather than on probabilities (Brandstätter \& Kühberger, 2005; Deane, 1969; Loewenstein et al., 2001; Sunstein, 2003), and it is based on the sequential structure of the Take The Best heuristic (Gigerenzer \& Goldstein, 1996), which is a heuristic for inferences, whereas the priority heuristic is a model of preferential choices. Finally, the priority heuristic incorporates aspiration levels into its choice algorithm (Luce, 1956; Simon, 1983). The generalization of the priority heuristic to nonpositive prospects (all outcomes are negative or zero) is straightforward. The heuristic is identical except that "gains" are replaced by "losses":

Priority Rule. Go through reasons in the order: minimum loss, probability of minimum loss, maximum loss.

Stopping Rule. Stop examination if the minimum losses differ by $1 / 10$ (or more) of the maximum loss; otherwise, stop examination if probabilities differ by $1 / 10$ (or more) of the probability scale.

Decision Rule. Choose the gamble with the more attractive loss (probability).

The term attractive refers to the gamble with the lower (minimum or maximum) loss and the higher probability of the minimum loss

Next, we generalize the heuristic to gambles with more than two outcomes (assuming nonnegative prospects):

Priority Rule. Go through reasons in the order: minimum gain, probability of minimum gain, maximum gain, probability of maximum gain.

Stopping Rule. Stop examination if the gains differ by $1 / 10$ (or more) of the maximum gain; otherwise, stop examination if probabilities differ by $1 / 10$ (or more) of the probability scale.

Decision Rule. Choose the gamble with the more attractive gain (probability).

This priority rule is identical with that for the two-outcome gambles, apart from the addition of a fourth reason. In gambles with more than two outcomes, the probability of the maximum outcome is informative because it is no longer the logical complement of the probability of the minimum outcome. The stopping rule is also 
identical, except for the fact that the maximum gain is no longer the last reason, and therefore the same aspiration levels apply to both minimum and maximum gains. The decision rule is identical with that for the two-outcome case. Finally, the algorithm is identical for gains and losses, except that "gains" are replaced by "losses."

The priority heuristic is simple in several respects. It typically consults only one or a few reasons; even if all are screened, it bases its choice on only one reason. Probabilities are treated as linear, and a 1/10 aspiration level is used for all reasons except the last, in which the amount of difference is ignored. No parameters for overweighting small probabilities and underweighting large probabilities or for the value function are built in. Can this simple model account for people's choices as well as multiparameter models can? To answer this question, we test whether the priority heuristic can accomplish the following:

1. Account for evidence at variance with expected utility theory, namely (a) the Allais paradox, (b) risk aversion for gains if probabilities are high, (c) risk seeking for gains if probabilities are low (e.g., lottery tickets), (d) risk aversion for losses if probabilities are low (e.g., buying insurance), (e) risk seeking for losses if probabilities are high, (f) the certainty effect, (g) the possibility effect, and (h) intransitivities; and

2. Predict the empirical choices in four classes of problems: (a) simple choice problems (no more than two nonzero outcomes; Kahneman \& Tversky, 1979), (b) problems involving multiple-outcome gambles (Lopes \& Oden, 1999), (c) problems inferred from certainty equivalents (Tversky \& Kahneman, 1992), and (d) problems involving randomly sampled gambles (Erev, Roth, Slonim, \& Barron, 2002).

Can the Priority Heuristic Predict Violations of Expected Utility Theory?

\section{The Allais Paradox}

In the early 1950 s, choice problems were proposed that challenged expected utility theory as a descriptive framework for risky choice (Allais, 1953, 1979). For instance, according to the independence axiom of expected utility, aspects that are common to both gambles should not influence choice behavior (Savage, 1954; von Neumann \& Morgenstern, 1947). For any three alternatives $X$, $Y$, and $Z$, the independence axiom can be written (Fishburn, 1979):

$$
\text { If } p X+(1-p) Z>p Y+(1-p) Z \text {, then } X>Y
$$

The following choice problems produce violations of the axiom (Allais, 1953, p. 527):

$$
\begin{aligned}
& \text { A: } \quad 100 \text { million } \quad p=1.00 \\
& \text { B: } \quad 500 \text { million } \quad p=.10 \\
& 100 \text { million } \quad p=.89 \\
& 0 \quad p=.01
\end{aligned}
$$

By eliminating a .89 probability to win 100 million from both $A$ and $B$, Allais obtained the following gambles:

$$
\begin{array}{lll}
\text { C: } & 100 \text { million } & \\
& 0 & p=.11 \\
\text { D: } & 500 \text { million } & p=.89 \\
& 0 & p=.90 .
\end{array}
$$

The majority of people chose $A$ over $B$, and $D$ over $C$ (MacCrimmon, 1968), which constitutes a violation of the axiom.

Expected utility does not predict whether $A$ or $B$ will be chosen; it only makes predictions of the type "if $A$ is chosen over $B$, then it follows that $C$ is chosen over $D$." The priority heuristic, in contrast, makes stronger predictions: It predicts whether $A$ or $B$ is chosen, and whether $C$ or $D$ is chosen. Consider the choice between $A$ and $B$. The maximum payoff is 500 million, and therefore the aspiration level is 50 million; 100 million and 0 represent the minimum gains. Because the difference (100 million) exceeds the aspiration level of 50 million, the minimum gain of 100 million is considered good enough, and people are predicted to select the sure gain $A$. That is, the heuristic predicts the majority choice correctly.

In the second choice problem, the minimum gains ( 0 and 0$)$ do not differ. Hence, the probabilities of the minimum gains are attended to, $p=.89$ and .90 , a difference that falls short of the aspiration level. The higher maximum gain (500 million vs. 100 million) thus decides choice, and the prediction is that people will select gamble $D$. Again, this prediction is consistent with the choice of the majority. Together, the pair of predictions amounts to the Allais paradox.

The priority heuristic captures the Allais paradox by using the heuristic building blocks of order, a stopping rule with a $1 / 10$ aspiration level, a lexicographic decision rule, and the tendency to avoid the worst possible outcome.

\section{The Reflection Effect}

The reflection effect refers to the empirically observed phenomenon that preferences tend to reverse when the sign of the outcomes is changed (Fishburn \& Kochenberger, 1979; Markowitz, 1952; Williams, 1966). Rachlinski's (1996) copyright litigation problem offers an illustration in the context of legal decision making. Here, the choice is between two gains or between two losses for the plaintiff and defendant, respectively:

The plaintiff can either accept a $\$ 200,000$ settlement [*] or face a trial with a .50 probability of winning $\$ 400,000$, otherwise nothing.

The defendant can either pay a $\$ 200,000$ settlement to the plaintiff or face a trial with a .50 probability of losing $\$ 400,000$, otherwise nothing [*]

The asterisks in brackets indicate which alternative the majority of law students chose, depending on whether they were cast in the role of the plaintiff or the defendant. Note that the two groups made opposite choices. Assuming that plaintiffs used the priority heuristic, they would have first considered the minimum gains, $\$ 200,000$ and $\$ 0$. Because the difference between the minimum gains is larger than the aspiration level $(\$ 40,000$ rounded to the next prominent number, $\$ 50,000)$, plaintiffs would have stopped examination and chosen the alternative with the more attractive minimum gain, that is, the settlement. The plaintiff's gain is the defendant's loss: Assuming that defendants also used the priority 
heuristic, they would have first considered the minimum losses, which are $\$ 200,000$ and $\$ 0$. Again, because the difference between these outcomes exceeds the aspiration level, defendants would have stopped examination and chosen the alternative with the more attractive minimum loss, that is, the trial. In both cases, the heuristic predicts the majority choice.

How is it possible that the priority heuristic predicts the reflection effect without - as prospect theory does-introducing value functions that are concave for gains and convex for losses? In the gain domain, the minimum gains are considered first, thus implying risk aversion. In the loss domain, the minimum losses are considered first, thus implying risk seeking. Risk aversion for gains and risk seeking for losses together make up the reflection effect.

\section{The Certainty Effect}

According to Allais (1979), the certainty effect captures people's "preference for security in the neighborhood of certainty" ( $p$. 441). A simple demonstration is the following (Kahneman \& Tversky, 1979):

$$
\begin{array}{lll}
\text { A: } & 4,000 \text { with } & \\
& 0 \text { with } & p=.80 \\
\text { B: } & 3,000 \text { with } & p=1.00
\end{array}
$$

\begin{tabular}{|c|c|}
\hline$C:$ & 4,000 with \\
\hline & 0 with \\
\hline$D:$ & 3,000 with \\
\hline & 0 with \\
\hline
\end{tabular}

A majority of people $(80 \%)$ selected the certain alternative $B$.

Now the majority of people $(65 \%)$ selected gamble $C$ over $D$. According to expected utility theory, the choice of $B$ implies that $u(3,000) / u(4,000)>4 / 5$, whereas the choice of $C$ implies the reverse inequality.

The priority heuristic starts by comparing the minimum gains of the alternatives $A(0)$ and $B(3,000)$. The difference exceeds the aspiration level of 500 (400, rounded to the next prominent number); examination is stopped; and the model predicts that people prefer the sure gain $B$, which is in fact the majority choice. Between $C$ and $D$, the minimum gains ( 0 and 0 ) do not differ; in the next step, the heuristic compares the probabilities of the minimum gains (.80 and .75). Because this difference does not reach 10 percentage points, the decision is with the higher maximum gain, that is, gamble $C$ determines the decision.

As the example illustrates, it is not always the first reason (minimum gain) that determines choice; it can also be one of the others. The priority heuristic can predict the certainty effect without assuming a specific probability weighting function.

\section{The Possibility Effect}

\begin{tabular}{|c|c|c|}
\hline \multirow[t]{2}{*}{$A:$} & 6,000 with & $p=.45$ \\
\hline & 0 with & $p=.55$ \\
\hline \multirow[t]{2}{*}{$B:$} & 3,000 with & $p=.90$ \\
\hline & 0 with & $p=.10$ \\
\hline
\end{tabular}

To demonstrate the possibility effect, participants received the following two choice problems (Kahneman \& Tversky, 1979):
The majority of people (86\%) selected gamble $B$.

$$
\begin{aligned}
& \text { C: } \quad 6,000 \text { with } \quad p=.001 \\
& 0 \text { with } \quad p=.999 \\
& \text { D: } \quad 3,000 \text { with } \quad p=.002 \\
& 0 \text { with } \quad p=.998
\end{aligned}
$$

In the second problem, most people $(73 \%)$ chose gamble $C$. This problem is derived from the first by multiplying the probabilities of the nonzero gains with $1 / 450$, making the probabilities of winning merely "possible." Note that in the certainty effect, "certain" probabilities are made "probable," whereas in the possibility effect, "probable" probabilities are made "possible." Can the priority heuristic predict this choice pattern?

In the first choice problem, the priority heuristic starts by comparing the minimum gains ( 0 and 0$)$. Because there is no difference, the probabilities of the minimum gains (.55 and .10) are examined. This difference exceeds 10 percentage points, and the priority heuristic, consistent with the majority choice, selects gamble $B$. Analogously, in the second choice problem, the minimum gains ( 0 and 0$)$ are the same; the difference between the probabilities of the minimum gains (.999 and .998) does not exceed 10 percentage points. Hence, the priority heuristic correctly predicts the choice of gamble $C$, because of its higher maximum gain of 6,000 .

\section{The Fourfold Pattern}

The fourfold pattern refers to the phenomenon that people are generally risk averse when the probability of winning is high but risk seeking when it is low (as when buying lotteries) and risk averse when the probability of losing is low (as with buying insurance) but risk seeking when it is high. Table 1 exemplifies the fourfold pattern (Tversky \& Fox, 1995).

Table 1 is based on certainty equivalents $C$ (obtained from choices rather than pricing). Certainty equivalents represent that amount of money where a person is indifferent between taking the risky gamble or the sure amount $C$. For instance, consider the first cell: The median certainty equivalent of $\$ 14$ exceeds the expected value of the gamble (\$5). Hence, in this case people are risk seeking, because they prefer the risky gamble over the sure gain of $\$ 5$. This logic applies in the same way to the other cells.

The certainty equivalent information of Table 1 directly lends itself to the construction of simple choice problems. For instance, from the first cell we obtain the following choice problem:

Table 1

The Fourfold Pattern

\begin{tabular}{lll}
\hline Probability & \multicolumn{1}{c}{ Gain } & \multicolumn{1}{c}{ Loss } \\
\hline Low & $C(100, .05)=14$ & $C(-100, .05)=-8$ \\
& Risk seeking & Risk aversion \\
High & $C(100, .95)=78$ & $C(-100, .95)=-84$ \\
& Risk aversion & Risk seeking \\
\hline
\end{tabular}

Note. $C(100, .05)$ represents the median certainty equivalent for the gamble to win $\$ 100$ with probability of .05 , otherwise nothing (based on Tversky \& Fox, 1995). 


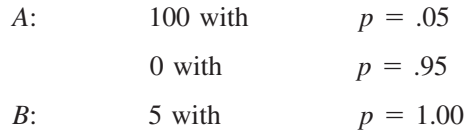

The priority heuristic starts by comparing the minimum gains $(0$ and 5). Because the sure gain of $\$ 5$ falls short of the aspiration level of $\$ 10$, probabilities are attended to. The probabilities of the minimum gains do not differ either $(1.00-.95<.10)$; hence, people are predicted to choose the risky gamble $A$, because of its higher maximum gain. This is in accordance with the certainty equivalent of $\$ 14$ (see Table 1), which implies risk seeking. Similarly, if the probability of winning is high, we obtain:

$$
\begin{array}{lll}
\text { A: } & 100 \text { with } & p=.95 \\
\text { B: } & 0 \text { with } & p=.05 \\
& 95 \text { with } & p=1.00
\end{array}
$$

Here, the sure gain of $\$ 95$ surpasses the aspiration level (\$10) and the priority heuristic predicts the selection of the sure gain $B$, which is in accordance with the risk-avoidant certainty equivalent in Table 1 $(\$ 78<\$ 95)$. The application to losses is straightforward:

$$
\begin{aligned}
& \text { A: } \quad-100 \text { with } \quad p=.05 \\
& \begin{aligned}
& 0 \text { with } & p & =.95 \\
B: & -5 \text { with } & p & =1.00
\end{aligned}
\end{aligned}
$$

Because the minimum losses $(0$ and -5$)$ do not differ, the probabilities of the minimum losses (.95 and 1.00) are attended to, which do not differ either. Consequently, people are predicted to choose the sure loss $B$, because of its lower maximum loss $(-5$ vs. -100$)$. This is in accordance with the risk-avoidant certainty equivalent in Table 1. Similarly, if the probability of losing is high we obtain:

$$
\begin{array}{lll}
\text { A: } & -100 \text { with } & p=.95 \\
\text { B: } & \text { 0 with } & p=.05 \\
& -95 \text { with } & p=1.00
\end{array}
$$

In this case, the minimum losses differ $(0-[-95]>10)$ and the priority heuristic predicts the selection of the risky gamble $A$, which corresponds to the certainty equivalent of Table 1 .

Note that in this last demonstration, probabilities are not attended to and one does not need to assume some nonlinear function of decision weights. As shown above, the priority heuristic correctly predicts the reflection effect, and consequently, the entire fourfold pattern in terms of one simple, coherent strategy.

\section{Intransitivities}

Intransitivities violate expected utility's fundamental transitivity axiom, which states that a rational decision maker who prefers $X$ to $Y$ and $Y$ to $Z$ must then prefer $X$ to $Z$ (von Neumann \& Morgenstern, 1947). Consider the choice pattern in Table 2, which shows the percentages of choices in which the row gamble was chosen over the column gamble. For instance, in $65 \%$ of the choices, gamble $A$ was chosen over gamble $B$. As shown therein, people prefer gambles $A>\mathrm{B}, \mathrm{B}>\mathrm{C}, \mathrm{C}>\mathrm{D}$, and $D>\mathrm{E}$. However, they violate transitivity by selecting gamble $E$ over $A$.

If one predicts the majority choices with the priority heuristic, one gets gamble $A>\mathrm{B}$ because the minimum gains are the same, their probabilities do not differ, and the maximum outcome of $A$ is
Table 2

Violations of Transitivity

\begin{tabular}{ccccc}
\hline Gamble & $B$ & $C$ & $D$ & $E$ \\
\hline$A(5.00, .29)$ & $\mathbf{. 6 5}$ & $\mathbf{. 6 8}$ & .51 & $\mathbf{. 3 7}$ \\
$B(4.75, .33)$ & - & $\mathbf{. 7 3}$ & $\mathbf{. 5 6}$ & $\mathbf{. 4 5}$ \\
$C(4.50, .38)$ & & - & $\mathbf{. 7 3}$ & $\mathbf{. 6 5}$ \\
$D(4.25, .42)$ & & & - & $\mathbf{. 7 5}$ \\
$E(4.00, .46)$ & & & & - \\
\hline
\end{tabular}

Note. Gamble $A(5.00, .29)$, for instance, offers a win of $\$ 5$ with probability of .29, otherwise nothing. Cell entries represent proportion of times that the row gamble was preferred to the column gamble, averaged over all participants from Tversky (1969). Bold numbers indicate majority choices correctly predicted by the priority heuristic.

higher. Similarly, the heuristic can predict all 10 majority choices with the exception of the .51 figure (a close call) in Table 2 . Note that the priority heuristic predicts gamble $A>\mathrm{B}, B>\mathrm{C}, C>\mathrm{D}$, $D>\mathrm{E}$, and $E>\mathrm{A}$, which results in the intransitive circle. In contrast, cumulative prospect theory, which reduces to prospect theory for these simple gambles, or the transfer-of-attentionexchange model attach a fixed overall value $V$ to each gamble and therefore cannot predict this intransitivity.

\section{Can the Priority Heuristic Predict Choices in Diverse Sets of Choice Problems?}

One objection to the previous demonstration is that the priority heuristic has been tested on a small set of choice problems, one for each anomaly. How does it fare when tested against a larger set of problems? We tested the priority heuristic in four different sets of choice problems (Erev et al., 2002; Kahneman \& Tversky, 1979; Lopes \& Oden, 1999; Tversky \& Kahneman, 1992). Two of these sets of problems were designed to test prospect theory and cumulative prospect theory, and one was designed to test securitypotential/aspiration theory (Lopes \& Oden, 1999); none, of course, were designed to test the priority heuristic. The contestants used were three modifications of expected utility theory: cumulative prospect theory, security-potential/aspiration theory, and the transfer-of-attention-exchange model (Birnbaum \& Chavez, 1997). In addition, we included the classic heuristics simulated by Thorngate (1980); the lexicographic and the equal-weight heuristic (Dawes, 1979) from Payne et al. (1993); and the tallying heuristic (see Table 3). The criterion for each of the four sets of problems was to predict the majority choice. This allows a comparison between the various heuristics, as well as between heuristics, cumulative prospect theory, security-potential/aspiration theory, and the transfer-of-attention-exchange model.

\section{The Contestants}

The contesting heuristics can be separated into two categories: those that use solely outcome information and ignore probabilities altogether (outcome heuristics) and those that use at least rudimentary probabilities (dual heuristics). ${ }^{2}$ These heuristics are de-

\footnotetext{
${ }^{2}$ We did not consider three of the heuristics listed by Thorngate (1980). These are low expected payoff elimination, minimax regret, and low payoff elimination. These strategies require extensive computations.
} 
Table 3

Heuristics for Risky Choice

Outcome heuristics

Equiprobable: Calculate the arithmetic mean of all monetary outcomes within a gamble. Choose the gamble with the highest monetary average.

Prediction: Equiprobable chooses $B$, because $B$ has a higher mean $(3,000)$ than $A(2,000)$.

Equal-weight: Calculate the sum of all monetary outcomes within a gamble. Choose the gamble with the highest monetary sum.

Prediction: Equal-weight chooses $A$, because $A$ has a higher sum $(4,000)$ than $B(3,000)$.

Minimax: Select the gamble with highest minimum payoff.

Prediction: Minimax chooses $B$, because $A$ has a lower minimum outcome $(0)$ than $B(3,000)$.

Maximax: Choose the gamble with the highest monetary payoff.

Prediction: Maximax chooses A, because its maximum payoff $(4,000)$ is the highest outcome.

Better-than-average: Calculate the grand average of all outcomes from all gambles. For each gamble, count the number of outcomes equal to or above the grand average. Then select the gamble with the highest number of such outcomes.

Prediction: The grand average equals $7,000 / 3=2,333$. Because both $A$ and $B$ have one outcome above this threshold, the better-than-average heuristic has to guess.

Dual heuristics

Tallying: Give a tally mark to the gamble with (a) the higher minimum gain, (b) the higher maximum gain, (c) the lower probability of the minimum gain, and (d) the higher probability of the maximum gain. For losses, replace "gain" by "loss" and "higher" by "lower" (and vice versa). Select the gamble with the higher number of tally marks.

Prediction: Tallying has to guess, because both $B$ (one tally mark for the higher minimal outcome, one for the higher probability of the maximum outcome) and $A$ (one tally mark for the lower probability of the minimal outcome, one for the higher maximum outcome) receive two tally marks each.

Most-likely: Determine the most likely outcome of each gamble and their respective payoffs. Then select the gamble with the highest, most likely payoff.

Prediction: Most-likely selects 4,000 as the most likely outcome for $A$ and 3,000 as the most likely outcome for $B$. Most-likely chooses $A$, because 4,000 exceeds 3,000.

Lexicographic: Determine the most likely outcome of each gamble and their respective payoffs. Then select the gamble with the highest, most likely payoff. If both payoffs are equal, determine the second most likely outcome of each gamble, and select the gamble with the highest (second most likely) payoff. Proceed until a decision is reached.

Prediction: Lexicographic selects 4,000 as the most likely outcome for $A$ and 3,000 as the most likely outcome for $B$. Lexicographic chooses $A$, because 4,000 exceeds 3,000.

Least-likely: Identify each gamble's worst payoff. Then select the gamble with the lowest probability of the worst payoff.

Prediction: Least-likely selects 0 as the worst outcome for $A$ and 3,000 as the worst outcome for $B$. Leastlikely chooses $A$, because 0 is less likely to occur (i.e., with $p=.20)$ than $3,000(p=1.00)$.

Probable: Categorize probabilities as "probable" (i.e., $p \geq .50$ for a two-outcome gamble, $p \geq .33$ for a three-outcome gamble, etc.) or "improbable." Cancel improbable outcomes. Then calculate the arithmetic mean of the probable outcomes for each gamble. Finally, select the gamble with the highest average payoff.

Prediction: Probable chooses $A$, because of its higher probable outcome $(4,000)$ compared with $B(3,000)$.

Note. Heuristics are from Thorngate (1980) and Payne et al. (1993). The prediction for each heuristic refers to the choice between $A(4,000, .80)$ and $B(3,000)$.

fined in Table 3, in which their algorithm is explained through the following choice problem:

A:

$80 \%$ chance to win 4,000

$20 \%$ chance to win 0

$B: \quad 3,000$ for sure
Cumulative prospect theory (Tversky \& Kahneman, 1992) attaches decision weights to cumulated rather than single probabilities. The theory uses five adjustable parameters. Three parameters fit the shape of the value function; the other two fit the shape of the probability weighting function. The value function is 


$$
\begin{gathered}
v(x)=x^{\alpha} \text { if } x \geq 0, \text { and } \\
v(x)=-\lambda(-x)^{\beta} \text { if } x<0 .
\end{gathered}
$$

The $\alpha$ and $\beta$ parameters modulate the curvature for the gain and loss domain, respectively; the $\lambda$ parameter $(\lambda>1)$ models loss aversion. The weighting function is:

$$
\begin{gathered}
w^{+}(p)=p^{\gamma} /\left(p^{\gamma}+(1-p)^{\gamma}\right)^{1 / \gamma}, \text { and } \\
w^{-}(p)=p^{\delta} /\left(p^{\delta}+(1-p)^{\delta}\right)^{1 / \delta},
\end{gathered}
$$

where the $\gamma$ and $\delta$ parameters model the inverse $\mathrm{S}$ shape of the weighing function for gains and losses, respectively.

Another theory that incorporates thresholds (i.e., aspiration levels) in a theory of choice is security-potential/aspiration theory (Lopes, 1987, 1995; for details, see Lopes \& Oden, 1999). Security-potential/aspiration theory is a six-parameter theory, which integrates two logically and psychologically independent criteria. The security-potential criterion is based on a rank-dependent algorithm (Quiggin, 1982; Yaari, 1987) that combines outcomes and probabilities in a multiplicative way. The aspiration criterion is operationalized as the probability to obtain some previously specified outcome. Both criteria together enable security-potential/ aspiration theory to model people's choice behavior.

The third modification of expected utility theory entering the contests is the transfer-of-attention-exchange model (Birnbaum \& Chavez, 1997), which was proposed as a response to problems encountered by prospect theory and cumulative prospect theory. This model has three adjustable parameters and is a special case of the more general configural weight model (Birnbaum, 2004). Like prospect theory, the transfer-of-attention-exchange model emphasizes how choice problems are described and presented to people. Unlike prospect theory, it offers a formal theory to capture the effects of problem formulations on people's choice behavior.

In models with adjustable parameters, parameter estimates are usually fitted for a specific set of choice problems and individuals. Data fitting, however, comes with the risk of overfitting, that is, fitting noise (Roberts \& Pashler, 2000). To avoid this problem, we used the fitted parameter estimates from one set of choice problems to predict the choices in a different one. For cumulative prospect theory, we used three sets of parameter estimates from Erev et al. (2002); Lopes and Oden (1999) and Tversky and Kahneman (1992). For the choice problems by Kahneman and Tversky (1979), no such parameter estimates exist. The three sets of parameter estimates are shown in Table 4. As one can see, they cover a broad range of values. Thus, we could test the predictive power of cumulative prospect theory with three independent sets of parameter estimates for the Kahneman and Tversky (1979) choice problems, and with two independent sets of parameter estimates for each of the other three sets of problems. In addition, for testing security-potential/aspiration theory, we used the parameter estimates from Lopes and Oden (1999); for testing the transfer-of-attention-exchange model, we used its prior parameters (see Birnbaum, 2004), which were estimated from Tversky and Kahneman (1992), to predict choices for the other three sets of choice problems.
Table 4

Parameter Estimates for Cumulative Prospect Theory

\begin{tabular}{lcccccc}
\hline & \multicolumn{5}{c}{ Parameter estimates } \\
\cline { 2 - 7 } \multicolumn{1}{c}{ Set of problems } & $\alpha$ & $\beta$ & $\lambda$ & $\gamma$ & $\delta$ \\
\hline Erev et al. (2002) & 0.33 & & & 0.75 & \\
Lopes \& Oden (1999) & 0.55 & 0.97 & 1.00 & 0.70 & 0.99 \\
Tversky \& Kahneman (1992) & 0.88 & 0.88 & 2.25 & 0.61 & 0.69 \\
\hline
\end{tabular}

Note. The parameters $\alpha$ and $\beta$ capture the shape of the value function for gains and losses, respectively; $\lambda$ captures loss aversion; $\gamma$ and $\delta$ capture the shape of the probability weighting function for gains and losses, respectively. See Equations 5-8 in the text. The Erev et al. (2002) set of problems is based on gains only.

\section{Contest 1: Simple Choice Problems}

The first test set consisted of monetary one-stage choice prob4lems from Kahneman and Tversky (1979). ${ }^{3}$ These 14 choice problems were based on gambles of equal or similar expected value and contained no more than two nonzero outcomes.

Results. Figure 1 shows how well the heuristics, cumulative prospect theory, security-potential/aspiration theory, and the transfer-of-attention exchange model each predicted the majority response. The maximum number of correct predictions is 14 . The white parts of the columns show correct predictions due to guessing. All heuristics, with the exceptions of the priority, equiprobable, and the lexicographic heuristics, had to guess in this set of problems.

The priority heuristic predicted all 14 choice problems correctly. In no instance did it need to guess. All other heuristics performed at or near chance level, except for the equiprobable and tallying heuristics: Equiprobable correctly predicted 10 of 14, whereas tallying predicted 4 of 11 choices correctly. ${ }^{4}$ It is interesting that among the 10 heuristics investigated, those that used only outcome information performed slightly better than did those also using probability information.

For testing cumulative prospect theory, we used three different parameter sets. The first parameter set was from Lopes and Oden (1999) and resulted in 64\% correct predictions. The second set was from Tversky and Kahneman (1992) and resulted in 71\% correct predictions. The third was from Erev et al.'s (2002) randomly constructed gambles, which resulted in chance performance $(50 \%$ correct).

On average, cumulative prospect theory correctly predicted $64 \%$ of the majority choices. ${ }^{5}$ One might assume that each of the parameter sets failed in predicting the same choice problems. However, this was not the case; the failures to predict were

\footnotetext{
${ }^{3}$ These are the choice problems 1, 2, 3, 4, 7, 8, 3', 4', 7', 8', 13, 13', 14, $14^{\prime}$ in Kahneman and Tversky (1979).

${ }^{4}$ Note that tallying does not predict choice behavior for problems with more than two outcomes. Whereas it is easy to compare the highest and the lowest outcomes of each gamble as well as their respective probabilities, it is unclear how to evaluate the probabilities of an intermediate outcome.

${ }^{5}$ As one can see from Table 4, the Erev et al. (2002) estimates of prospect theory's parameters only refer to gains. Therefore, only a subset of the problems studied by Kahneman and Tversky (1979) could be predicted, which was accounted for by this and the following means.
} 


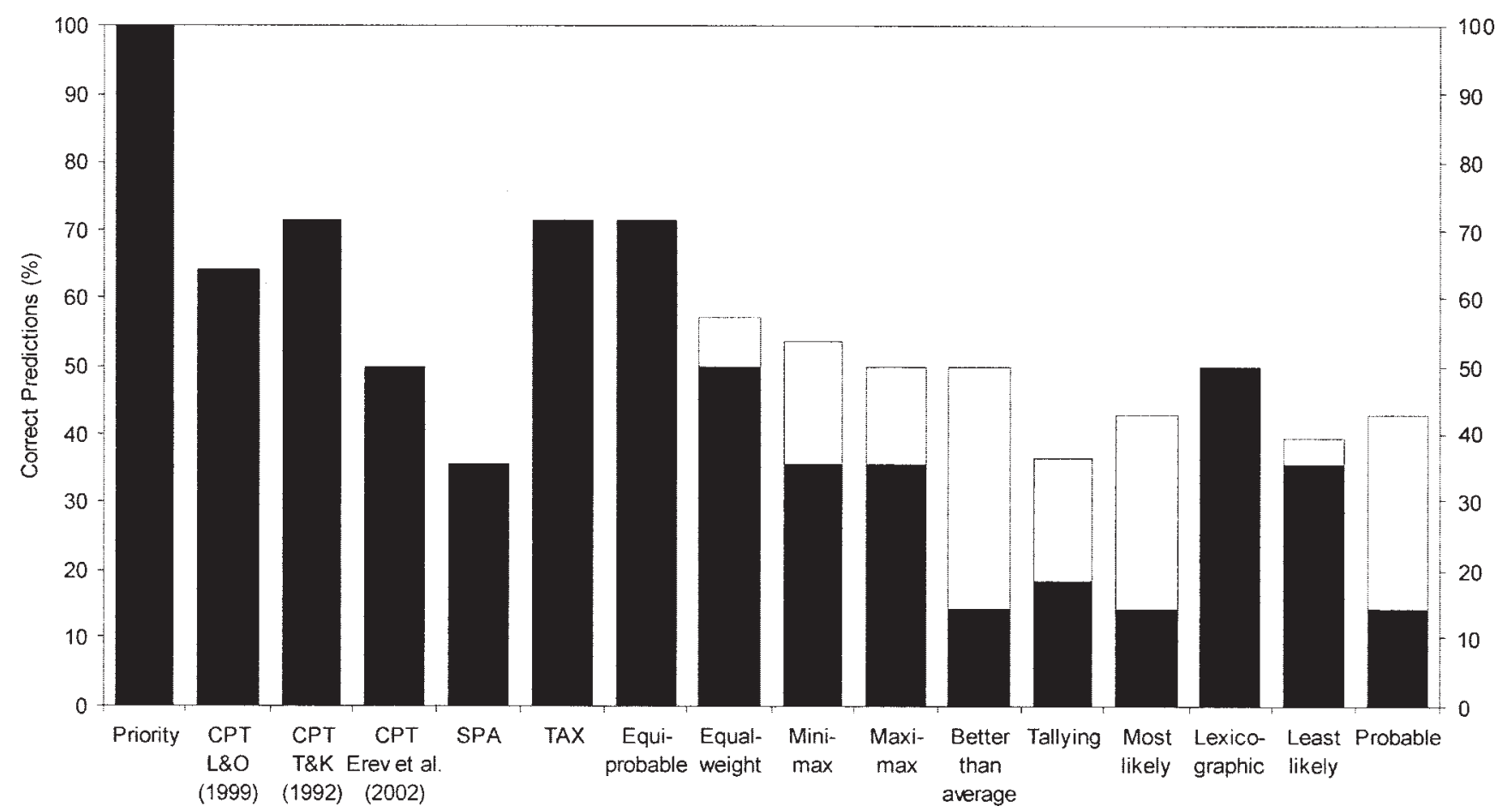

Figure 1. Correct predictions of the majority responses for all monetary one-stage choice problems (14) in Kahneman and Tversky (1979). The black parts of the bars represent correct predictions without guessing; the union of the black and white parts represents correct predictions with guessing (counting as 0.5). The Erev et al. (2002) set of problems consists of positive gambles; its fitted parameters allow only for predicting the choice behavior for positive one-stage gambles (making eight problems). Parameters for cumulative prospect theory (CPT) were estimated from Lopes and Oden (L\&O; 1999); Tversky and Kahneman (T\&K; 1992), and Erev et al., respectively. SPA $=$ security-potential/aspiration theory; TAX $=$ transfer-of-attention-exchange model.

distributed across 10 problems. This suggests that choice problems correctly predicted by one parameter set were incorrectly predicted by another set and vice versa. Finally, security-potential/aspiration theory correctly predicted 5 of 14 choice problems, which resulted in $36 \%$ correct predictions, and the transfer-of-attention-exchange model correctly predicted $71 \%$ of the choice problems (i.e., 10 of 14).

Why did the heuristics in Table 3 perform so dismally in predicting people's deviations from expected utility theory? Like the priority heuristic, these heuristics ignore information. However, the difference lies in how information is ignored.

For gains, the priority heuristic uses the same first reason that minimax does (see Table 3). Unlike minimax, however, the priority heuristic does not always base its choice on the minimum outcomes, but only when the difference between the minimum outcomes exceeds the aspiration level. If not, then the second reason, the probability of the minimum outcome, is given priority. This reason captures the policy of the least-likely heuristic (see Table 3). Again, the priority heuristic uses an aspiration level to "judge" whether this policy is reasonable. If not, the maximum outcome will decide, which is the policy of the maximax heuristic (see Table 3). The same argument holds for gambles with losses, except that the positions of minimax and maximax are switched. Thus, the sequential nature of the priority heuristic integrates several of the classic heuristics, brings them into a specific order, and uses aspiration levels to judge whether they apply.
In summary, the priority heuristic was able to predict the majority choice in all 14 choice problems in Kahneman and Tversky (1979). The other heuristics did not predict well, mostly at chance level, and cumulative prospect theory did best when its parameter values were estimated from Tversky and Kahneman (1992).

\section{Contest 2: Multiple-Outcome Gambles}

The fact that the priority heuristic can predict the choices in two-outcome gambles does not imply that it can do the same for multiple-outcome gambles. These are a different story, as illustrated by prospect theory (unlike the revised cumulative version), which encountered problems when it was applied to gambles with more than two nonzero outcomes. Consider the choice between the multiple-outcome gamble $A$ and the sure gain $B$ :

$\begin{array}{lll}\text { A: } & 0 \text { with } & p=.05 \\ & 10 \text { with } & p=.05 \\ 20 \text { with } & p=.05 \\ \ldots & & \\ & 190 \text { with } & p=.05 \\ B: & 95 \text { with } & p=1.00\end{array}$

The expected values of $A$ and $B$ are 95. According to the probability weighting function in prospect theory, each monetary out- 
come in gamble $A$ is overweighted, because $\pi(.05)>.05$. For the common value functions, prospect theory predicts a higher subjective value for the risky gamble $A$ than for the sure gain of 95 . In contrast, 28 of 30 participants opted for the sure gain $B$ (Brandstätter, 2004).

The priority heuristic gives first priority to the minimum outcomes, which are 0 and 95. The difference between these two values is larger than the aspiration level (20, because 19 is rounded to 20), so no other reason is examined and the sure gain is chosen.

The second set of problems consists of 90 pairs of five-outcome lotteries from Lopes and Oden (1999). In this set, the expected values of each pair are always similar or equal. The probability distributions over the five rank-ordered gains have six different shapes: Lotteries were (a) nonrisk (the lowest gain was larger than zero and occurred with the highest probability of winning), (b) peaked (moderate gains occurred with the highest probability of winning), (c) negatively skewed (the largest gain occurred with the highest probability of winning), (d) rectangular (all five gains were tied to the same probability, $p=.20$ ), (e) bimodal (extreme gains occurred with the highest probability of winning), and (f) positively skewed (the largest gain occurred with the lowest probability of winning). An example is shown in Figure 2.

These six gambles yielded 15 different choice problems. From these, Lopes and Oden (1999) created two other choice sets by (a) adding $\$ 50$ to each outcome and (b) multiplying each outcome by 1.145 , making $45(3 \times 15)$ choice problems. In addition, negative lotteries were created by appending a minus sign to the outcomes of the three positive sets, making 90 choice problems. This procedure yielded six different choice sets (standard, shifted, multiplied-separately for gains and losses), each one comprising all possible choices within a set (i.e., 15).

Results. The priority heuristic yielded $87 \%$ correct predictions, as shown in Figure 3. All other heuristics performed around chance level or below. The result from the previous competitionthat outcome heuristics are better predictors than the dual heuristics-did not generalize to multiple-outcome gambles.

The parameter values for cumulative prospect theory were estimated from two independent sets of problems. With the parameter estimates from the Tversky and Kahneman (1992) set of problems, cumulative prospect theory predicted $67 \%$ of the majority responses correctly. With the estimates from the Erev et al. (2002) set of problems, the proportion of correct predictions was $87 \%$. With the second set of parameter estimates, cumulative prospect theory tied with the priority heuristic, whereas cumulative prospect theory's performance was lower with the first set. Its average predictive accuracy was $73 \%$. The fact that it did not perform better than the heuristic did is somewhat surprising, given that cumulative prospect theory was specifically designed for multiple-outcome gambles. Finally, the transfer-of-attentionexchange model correctly predicted $63 \%$ of the majority responses.

Lopes and Oden (1999) fitted cumulative prospect theory to their set of problems. We used these parameter estimates and "tested" cumulative prospect theory on the Lopes and Oden set of problems, which is known as "data fitting." The resulting fitting power with five adjustable parameters was $87 \%$. A slightly higher result emerged for security-potential/aspiration theory, for which the fitting power with six parameters was $91 \%$.

To sum up, the 90 five-outcome problems no longer allowed the priority heuristic to predict $100 \%$ correctly. Nevertheless, the consistent result in the first two contests was that the priority heuristic could predict the majority response as well as or better than the three modifications of expected utility theory or any of the other heuristics. We were surprised by the heuristic's good performance, given that it ignores all intermediate outcomes and their probabilities. It is no doubt possible that gambles can be deliberately constructed with intermediate outcomes that the priority heuristic does not predict as well. Yet in these six systematically varied sets of gambles, no other model outperformed the priority heuristic.

\section{Contest 3: Risky Choices Inferred From Certainty Equivalents}

The previous analyses used the same kind of data, namely choices between explicitly stated gambles. The next contest introduces choices inferred from certainty equivalents. The certainty equivalent, $C$, of a risky gamble is defined as the sure amount of money $C$, where a person has no preference between the gamble and the sure amount. Certainty equivalents can be translated into choices between a risky gamble and a sure payoff. Our third test set comprised 56 gambles studied by Tversky and Kahneman (1992). These risky gambles are not a random or representative set of gambles. They were designed for the purpose of demonstrating that cumulative prospect theory accounts for deviations from expected utility theory. Half of the gambles are in the gain domain

\begin{tabular}{|c|c|c|c|}
\hline \multicolumn{3}{|c|}{ Short Shot } & Long Shot \\
\hline$\$ 140$ & |||||||||||||||||||||||||||||||||||||||| & $\$ 348$ & IIII \\
\hline$\$ 105$ & ||I||||||||||||||||||||||||| & $\$ 261$ & ||$I||||||||$ \\
\hline$\$ 70$ & $|\|||||||||||||||| \mid$ & $\$ 174$ & |||||||||||||||||| \\
\hline$\$ 35$ & \|\|\|\|\|\|$\|$ & $\$ 87$ & |||||||||||||||||||||||||||||| \\
\hline$\$ 0$ & IIII & $\$ 0$ & 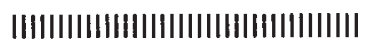 \\
\hline
\end{tabular}

Figure 2. A typical choice problem used in Contest 2, from Lopes and Oden (1999). Each lottery has 100 tickets (represented by marks) and has an expected value of approximately $\$ 100$. Values at the left represent gains or losses. Reprinted from Journal of Mathematical Psychology, 43, L. L. Lopes \& G. C. Oden, "The role of aspiration level in risky choice: A comparison of cumulative prospect theory and SP/A theory," p. 293. Copyright 1999 with permission from Elsevier. 


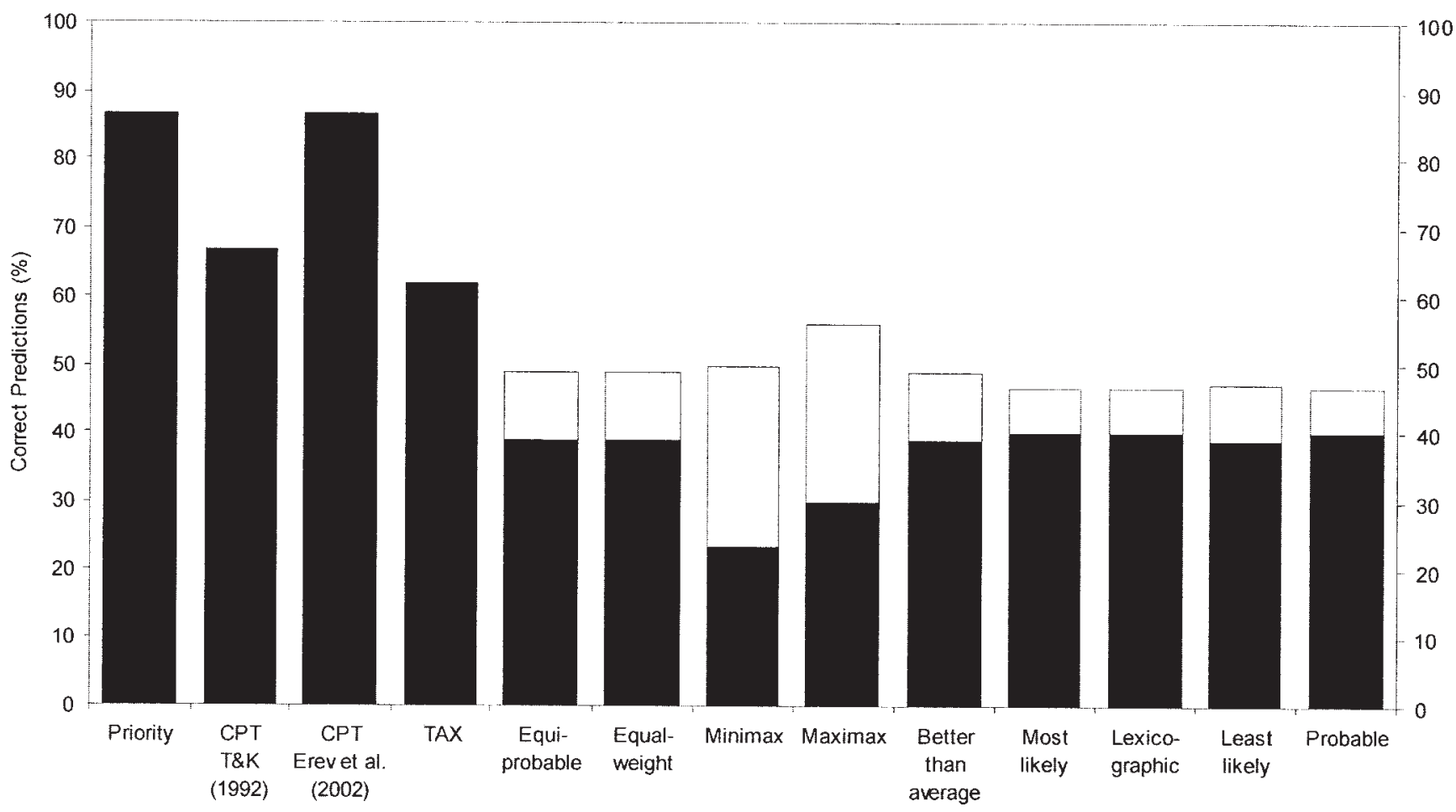

Figure 3. Correct predictions of the majority responses for the 90 five-outcome choice problems in Lopes and Oden (1999). The black parts of the bars represent correct predictions without guessing; the union of the black and white parts represents correct predictions with guessing (counting as 0.5). Tallying was not applicable (see Footnote 4). The parameters taken from Erev et al. (2002) predict gains only. Parameters for cumulative prospect theory (CPT) are from Tversky and Kahneman (T\&K; 1992) and Erev et al., respectively. TAX = transfer-ofattention-exchange model.

( $\$ x \geq 0$ ); for the other half, a minus sign was added. Each certainty equivalent was computed from observed choices (for a detailed description, see Brandstätter, Kühberger, \& Schneider, 2002). Consider a typical example from this set of problems:

$$
C(\$ 50, .10 ; \$ 100, .90)=\$ 83
$$

Because this empirical certainty equivalent falls short of the expected value of the gamble (\$95), people are called risk averse. We can represent this information as a choice between the risky gamble and a sure gain of equal expected value:

$$
\begin{array}{ll}
\text { A: } & 10 \% \text { chance to win } 50 \\
& 90 \% \text { chance to win } 100 \\
B: & 95 \text { for sure. }
\end{array}
$$

The priority heuristic predicts that the minimum outcomes, which are $\$ 50$ and $\$ 95$, are compared first. The difference between these two values is larger than the aspiration level $(\$ 10)$. No other reason is examined and the sure gain is chosen.

Results. The priority heuristic made $89 \%$ correct predictions (see Figure 4). The equiprobable heuristic was the second-best heuristic, with $79 \%$, followed by the better-than-average heuristic. All other heuristics performed at chance level or below, and tallying had to guess all the time (see Table 3 ). The pattern obtained resembles that of the first competition; the outcome heuristics fared better than did those that also used probability information.

Cumulative prospect theory achieved $80 \%$ correct predictions with the parameter estimates from the Lopes and Oden (1999) set of problems, and $75 \%$ with the Erev et al. (2002) data set (see Figure 4). Thus, the average predictive accuracy was $79 \%$. Security-potential/aspiration theory fell slightly short of these numbers and yielded $73 \%$ correct forecasts. In contrast, when one "tests" cumulative prospect theory on the same data (Tversky \& Kahneman, 1992) from which the five parameters were derived (i.e., data fitting rather than prediction), one can correctly "predict" $91 \%$ of the majority choices. The parameters of the transfer-of-attentionexchange model were fitted by Birnbaum and Navarrete (1998) on the Tversky and Kahneman (1992) data; thus, we cannot test how well it predicts the data. In data fitting, it achieved 95\% correct "predictions."

\section{Contest 4: Randomly Drawn Two-Outcome Gambles}

The final contest involved 100 pairs of two-outcome gambles that were randomly drawn (Erev et al., 2002). Almost all minimum outcomes were zero. This set of problems handicapped the priority heuristic, given that it could rarely make use of its top-ranked reason. An example from this set is the following (units are points that correspond to cents): 


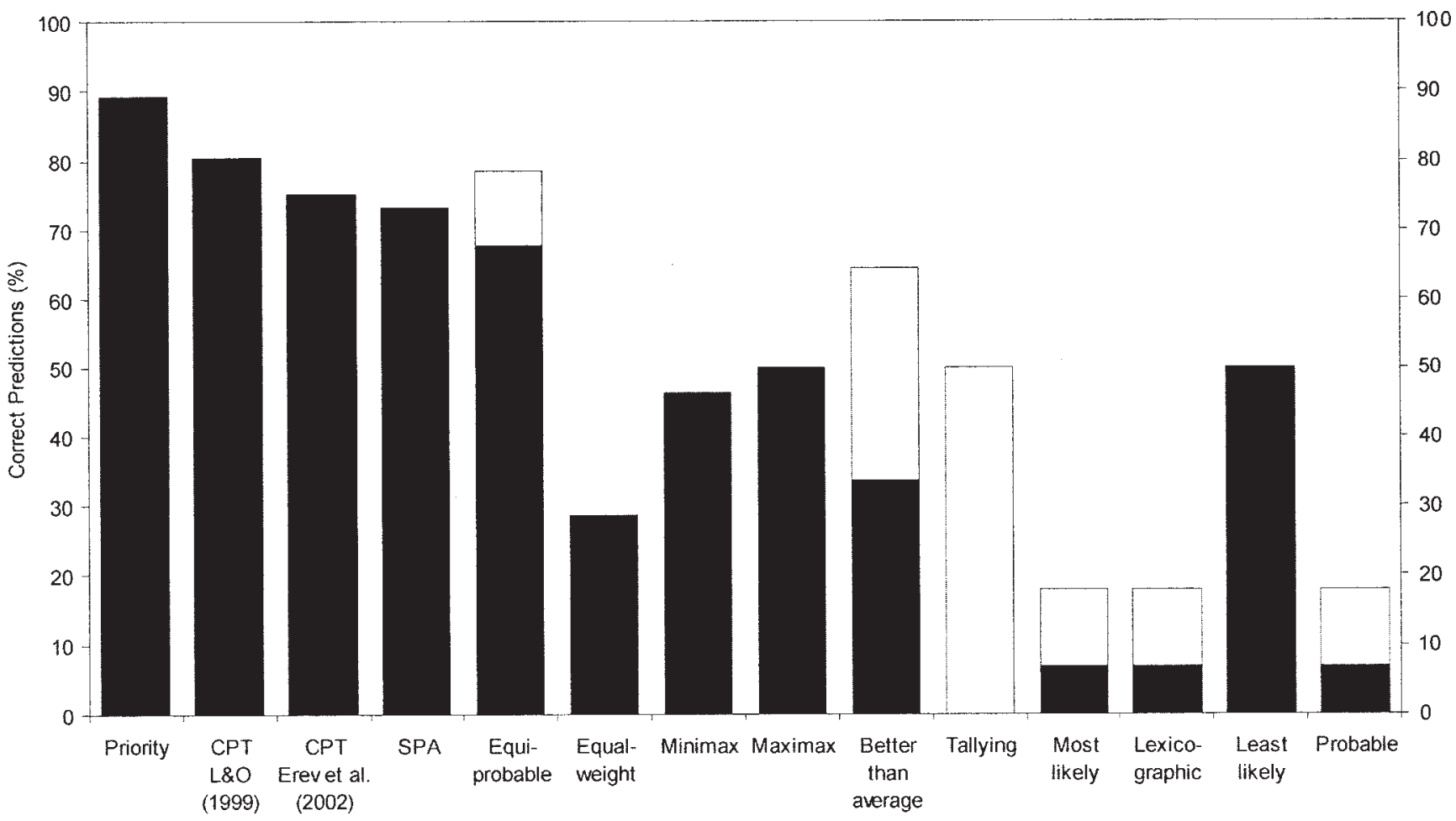

Figure 4. Correct predictions of the majority responses for the 56 certainty equivalence problems in Tversky and Kahneman (1992). The black parts of the bars represent correct predictions without guessing; the union of the black and white parts represents correct predictions with guessing (counting as 0.5 ). The parameters taken from Erev et al. (2002) predict gains only. Parameters for cumulative prospect theory (CPT) are from Lopes and Oden (L\&O; 1999) and Erev et al., respectively. In the latter set of problems, predictions refer to gains only. SPA $=$ security-potential/aspiration theory.

\section{A: $\quad 49 \%$ chance to win 77 \\ $51 \%$ chance to win 0 \\ B: $\quad 17 \%$ chance to win 98 \\ $83 \%$ chance to win 0}

These pairs of lotteries were created by random sampling of the four relevant values $x_{1}, p_{1}, x_{2}$, and $p_{2}$. Probabilities were randomly drawn from the uniform distribution $(.00, .01, .02, \ldots 1.00)$ and monetary gains from the uniform distribution $(1,2,3, \ldots 100)$. The constraint $\left(x_{1}-x_{2}\right)\left(p_{1}-p_{2}\right)<0$ eliminated trivial choices, and the sampling procedures generated choices consisting of gambles with unequal expected value.

Results. Although the priority heuristic could almost never use its top-ranked reason, it correctly predicted $85 \%$ of the majority choices reported by Erev et al. (2002). In this set, the outcome heuristics performed worse than those also using probability information did (see Figure 5). As a further consequence, the performance of minimax was near chance level, because its only reason, the minimum gains, was rarely informative, and it thus had to guess frequently (exceptions were four choice problems that included a sure gain). Cumulative prospect theory achieved $89 \%$ and $75 \%$ correct predictions, depending on the set of parameters, which resulted in an average of $82 \%$ correct predictions. The security-potential/aspiration theory correctly predicted $88 \%$, and the transfer of exchange model achieved $74 \%$ correct forecasts. In the four contests, with a total of nine tests of cumulative prospect theory, three tests of security-potential/aspiration theory, and three tests of the transfer-of-attention-exchange model, these $89 \%$ and $88 \%$ figures were the only instances in which the two models could predict slightly better than the priority heuristic did (for a tie, see Figure 3).

Again, we checked the fitting power of cumulative prospect theory by the Erev et al. (2002) set of problems. This resulted in a fitting power of $99 \%$. As in the previous analyses, a substantial discrepancy between fitting and prediction emerged.

\section{The Priority Heuristic as a Process Model}

Process models, unlike as-if models, can be tested on two levels: the choice and the process. In this article, we focus on how well the priority heuristic can predict choices, compared with competing theories. Yet we now want to illustrate how the heuristic lends itself to testable predictions concerning process. Recall that the priority heuristic assumes a sequential process of examining reasons that is stopped as soon as an aspiration level is met. Therefore, the heuristic predicts that the more reasons that people are required to examine, the more time they need for making a choice. Note that all three modifications of expected utility theory tested here (if interpreted as process models) assume that all pieces of information are used and thus do not imply this process prediction. 


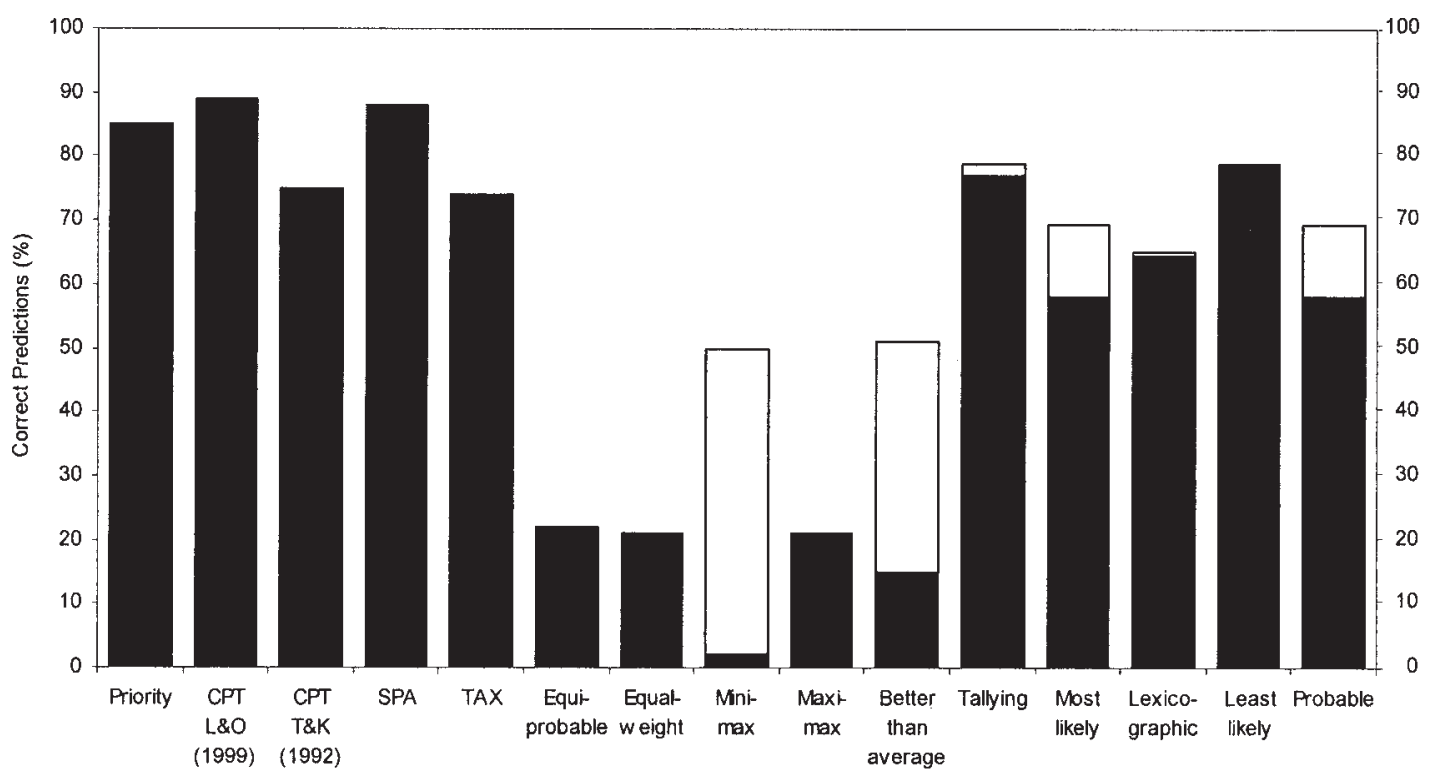

Figure 5. Correct predictions of the majority responses for the 100 random choice problems in Erev et al. (2002). The black parts of the bars represent correct predictions without guessing; the union of the black and white parts represents correct predictions with guessing (counting as 0.5). Parameters for cumulative prospect theory (CPT) are from Lopes and Oden (L\&O; 1999) and Tversky and Kahneman (T\&K; 1992), respectively. SPA $=$ security-potential/aspiration theory; TAX = transfer-of-attention-exchange model.

To illustrate the prediction, consider the following choice:

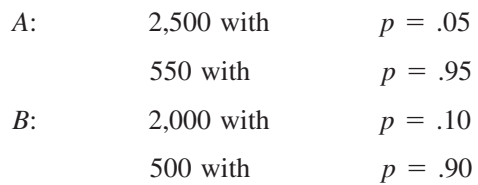

Given the choice between $A$ and $B$, the priority heuristic predicts that people examine three reasons and therefore need more time than for the choice between $C$ and $D$, which demands examining one reason only:

\begin{tabular}{|c|c|}
\hline$C:$ & 2,000 with \\
\hline & 500 with \\
\hline$D:$ & 2,000 with \\
\hline & 1,000 with \\
\hline
\end{tabular}

In summary, the prediction is as follows: If the priority heuristic implies that people examine more reasons (e.g., three as opposed to one), the measured time people need for responding will be longer. This prediction was tested in the following experiment for two-outcome gambles, for five-outcome gambles, for gains and losses, and for gambles of similar and dissimilar expected value.

Method. One hundred twenty-one students ( 61 females, 60 males; $M=$ 23.4 years, $S D=3.8$ years) from the University of Linz participated in this experiment. The experimental design was a 2 (one reason or three reasons examined) $\times 2$ (choice between 2 two-outcome gambles or choice between 2 five-outcome gambles) $\times 2$ (gambles of similar or dissimilar expected value) $\times 2$ (gains vs. losses) mixed-factorial design, with domain (gains vs. losses) as a between-participants factor and the other three manipulations as within-participants factors. The dependent variable, response time (in milliseconds), was measured from the first appearance of the decision problem until the moment when the participant indicated his or her choice by clicking either gamble $A$ or $B$. Then the next choice problem appeared on the computer screen. Each participant responded to 40 choice problems, which appeared in random order within each kind of set (i.e., two-outcome and five-outcome set). The order was counterbalanced so that half of the participants received the five-outcome gambles before the two-outcome gambles, whereas this order was reversed for the other half of the participants. All 40 choice problems from the gain domain (gains were converted into losses by adding a minus sign) are listed in the Appendix.

Results and discussion. The prediction was that the response time is shorter for those problems in which the priority heuristic implies that people stop examining after one reason, and it is longer when they examine all three reasons. As shown in Figure 6, results confirmed this prediction.

This result held for both choices between two-outcome gambles (one reason: $M d n=9.3, M=10.9, S E=0.20$; three reasons: $M d n=10.1, M=11.9, S E=0.21 ; z=-3.8, p=.001)$ and choices between five-outcome gambles (one reason: $M d n=10.3$, $M=12.6, S E=0.26$; three reasons: $M d n=11.8, M=14.1, S E=$ $0.41 ; z=-2.9, p=.004)$. It is not surprising that five-outcome gambles need more reading time than two-outcome gambles, which may explain the higher response time for the former. We additionally analyzed response times between the predicted number of reasons people examined (one or three) when the expected values were similar (one reason: $M d n=9.8, M=12.1, S E=0.24$; three reasons: $M d n=11.1, M=13.2, S E=0.30 ; z=-4.5, p=$ $.001)$ and when expected values were dissimilar (one reason: $M d n=9.7, M=11.5, S E=0.22$; three reasons: $M d n=10.1$, $M=12.1, S E=0.26 ; z=-1.7, p=.085)$; when people decided between two gains (one reason: $M d n=9.3, M=11.5, S E=0.22$; three reasons: $M d n=10.5, M=12.7, S E=0.27 ; z=-4.2, p=$ .001) and when they decided between two losses (one reason: 


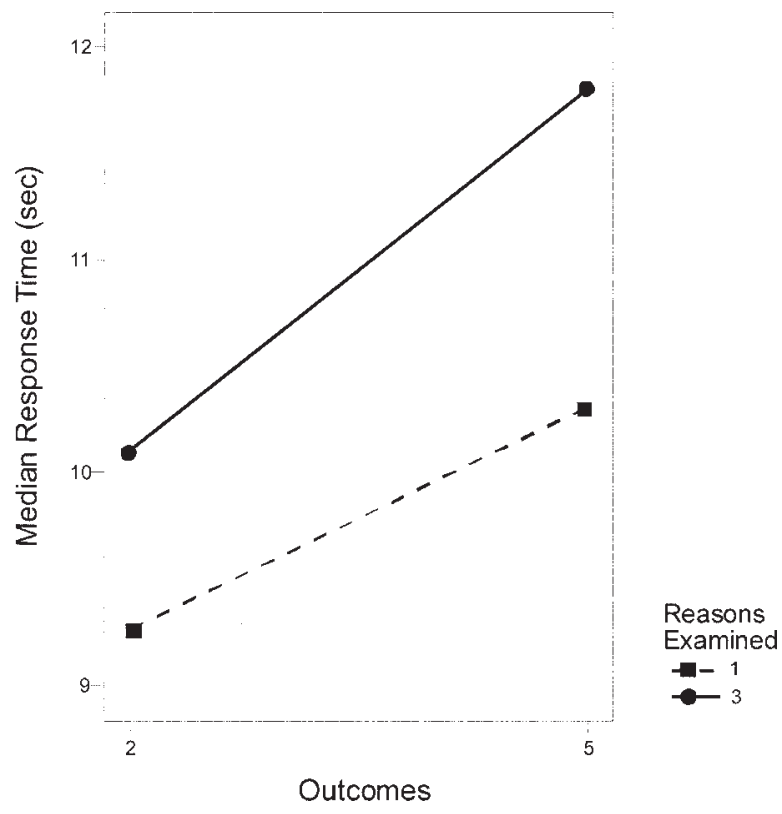

Figure 6. Participants' median response time dependent on the number of outcomes and the number of reasons examined.

$M d n=10.2, M=12.1, S E=0.25$; three reasons: $M d n=10.5$, $M=12.5, S E=0.29 ; z=-1.7, p=.086)$. In addition to our predictions, we observed that the effects are stronger for gambles from the gain domain than from the loss domain and when the expected values are similar rather than dissimilar.

The priority heuristic gives rise to process predictions that go beyond those investigated in this article. One of them concerns the order in which people examine reasons. Specifically, the priority heuristic predicts that reasons are considered in the following order: minimum gain, probability of minimum gain, and maximum gain. This and related predictions can be examined with processtracing methodologies such as eye tracking. Using mouse lab, for instance, Schkade and Johnson (1989) reported evidence for choice processes that are consistent with lexicographic strategies like the priority heuristic.

\section{Frugality}

Predictive accuracy is one criterion for comparing models of choice between gambles; frugality is another. The latter has not been the focus of models of risky choice. For instance, expected utility theory and cumulative prospect theory take all pieces of information into account (exceptions to this are sequential search models such as heuristics and decision field theory; see Busemeyer \& Townsend, 1993).

How to define frugality? All heuristics and modifications of expected utility theory assume a specific reading stage, in which all pieces of information are read and the relevant one (which varies from model to model) is identified. For instance, a person who relies on the minimax heuristic will read the text and determine what the minimal outcomes are. A person who relies on cumulative prospect theory will read the text and identify all relevant pieces of information from the point of view of this theory. This reading phase is common to all choice models and is not what we refer to in our definition of frugality. The frugality of a strategy refers to the processes that begin after the text is read.

We define frugality as the proportion of pieces of information that a model ignores when making a decision. Guessing, for instance, is the most frugal strategy; it ignores $100 \%$ of the information, and therefore its frugality is $100 \%$. In a two-outcome gamble, the probabilities are complementary, which reduces the number of pieces of information from eight to six (the two minimum outcomes, their probabilities, and the two maximum outcomes). Minimax, for instance, ignores four of these six pieces of information; thus its frugality is $67 \%$. The modifications of expected utility theory do not ignore any information (regardless of whether one assumes six or eight pieces of information), and thus their frugality is $0 \%$.

Unlike heuristics such as minimax, which always derive their decision from the same pieces of information, the frugality of the priority heuristic depends on the specific choice problem. For two-outcome gambles, the probabilities of the maximum outcomes are again complementary, reducing the number of pieces of information from eight to six. In making a choice, the priority heuristic then ignores either four pieces of information (i.e., the probabilities of the minimal outcomes and the maximal outcomes), two pieces of information (i.e., the maximal outcomes), or no information. This results in frugalities of $4 / 6,2 / 6$, and 0 , respectively. However, for the stopping rule, the heuristic needs information about the maximum gain (or loss), which reduces the frugalities to $3 / 6,1 / 6$, and 0 , respectively. ${ }^{6}$

For each of the four sets of choice problems, we calculated the priority heuristic's frugality score. In the first set of problems (see Figure 1; Kahneman \& Tversky, 1979), the priority heuristic ignored $22 \%$ of the information. For the five-outcome gambles in Figure 3, the heuristic ignored $78 \%$. As mentioned before, one reason for this is that the heuristic solely takes note of the minimum and maximum outcomes and their respective probabilities, and it ignores all other information. The modifications of expected utility theory, in contrast, ignored $0 \%$. In other words, for fiveoutcome gambles, the heuristic predicted people's choices $(87 \%)$ as good as or better than the modifications of expected utility theory with one fourth of the information. In the Tversky and Kahneman (1992) set of problems, the priority heuristic frugality score was $31 \%$; for the set of randomly chosen gambles, the heuristic ignored $15 \%$ of the information. This number is relatively low, because as mentioned before, the information about the minimum gain was almost never informative. In summary, the priority heuristic predicted the majority choice on the basis of fewer pieces of information than multiparameter models did, and its frugality depended strongly on the type of gamble in question.

\footnotetext{
${ }^{6}$ For two-outcome gambles, six instead of eight pieces of information yield a lower-bound estimate of the frugality advantage of the heuristics over parameter-based models such as cumulative prospect theory, which do not treat decision weights as complementary. For $n$-outcome gambles, with $n>2$, all $4 n$ pieces of information were used in calculating frugalities Similarly, in the case of ambiguity, we calculated a heuristic's frugality in a way to give this heuristic the best edge against the priority heuristic.
} 


\section{Overall Performance}

We now report the results for all 260 problems from the four contests. For each strategy, we calculated its mean frugality and the proportion of correct predictions (weighted by the number of choice problems per set of problems). As shown in Figure 7, there are three clusters of strategies: the modifications of expected utility and tallying, the classic choice heuristics, and the priority heuristic. The clusters have the following characteristics: The modifications of expected utility and tallying could predict choice fairly accurately but required the maximum amount of information. The classic heuristics were fairly frugal but performed dismally in predicting people's choices. The priority heuristic achieved the best predictive accuracy ( $87 \%$ ) while being relatively frugal.

Security-potential/aspiration theory, cumulative prospect theory, and the transfer-of-attention-exchange model correctly predicted $79 \%, 77 \%$, and $69 \%$ of the majority choices, respectively. With the exception of the least-likely heuristic and tallying, most classic heuristics did not predict better than chance. For instance, the performances of the minimax and lexicographic rules were $49 \%$ and $48 \%$, respectively.

The four sets of problems allowed for 15 comparisons between the predictive accuracy of the priority heuristic and cumulative prospect theory, security-potential/aspiration theory, and the transfer-of-attention-exchange model. $^{7}$ The priority heuristic

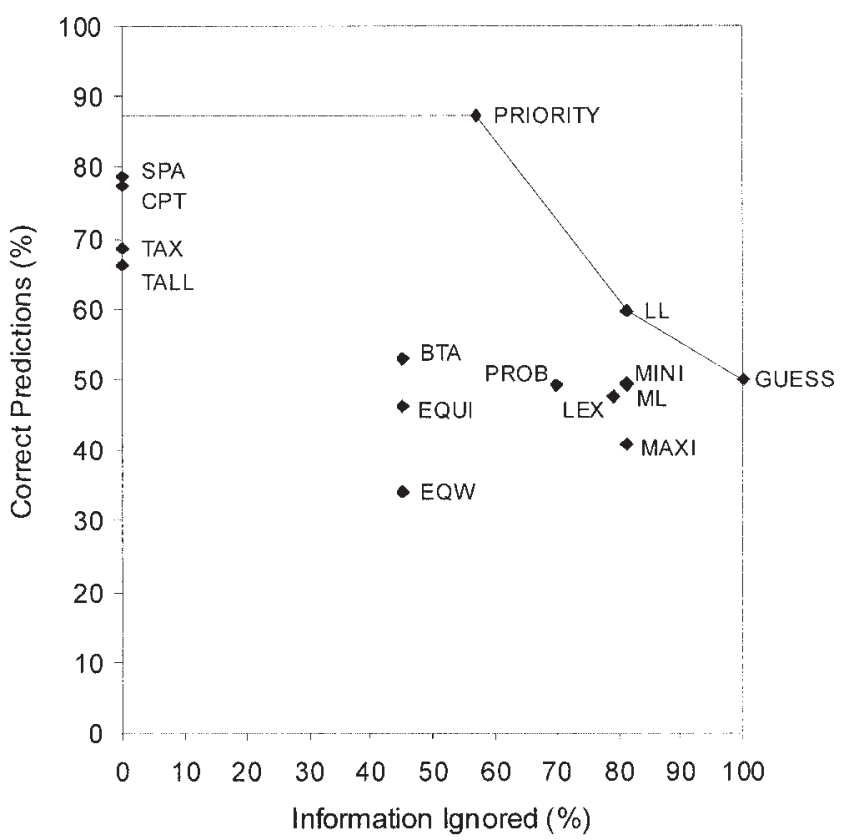

Figure 7. Predictability-frugality trade-off, averaged over all four sets of problems. The percentage of correct predictions refers to majority choices (including guessing). PRIORITY $=$ priority heuristic; SPA $=$ securitypotential/aspiration theory; $\mathrm{CPT}=$ cumulative prospect theory; $\mathrm{TAX}=$ transfer-of-attention-exchange model; TALL = tallying; $\mathrm{LL}=$ least-likely heuristic; BTA = better-than-average heuristic; $\mathrm{PROB}=$ probable heuristic; MINI = minimax heuristic; GUESS = pure guessing; EQUI = equiprobable heuristic; LEX = lexicographic heuristic; $\mathrm{ML}=$ most-likely heuristic; $\mathrm{MAXI}=\operatorname{maximax}$ heuristic; $\mathrm{EQW}=$ equal-weight heuristic. For a description of the heuristics, see Table 3. achieved the highest predictive accuracy in 12 of the 15 comparisons (Figures 1, 3, 4, and 5), and cumulative prospect theory and security-potential/aspiration theory in one case each (plus one tie).

\section{Discussion}

The present model of sequential choice continues the works of Luce (1956), Selten (2001), Simon (1957), and Tversky (1969). Luce (1956) began to model choice with a semiorder rule, and Tversky $(1969,1972)$ extended this work, adding heuristics such as "elimination by aspects." In his later work with Kahneman, he switched to modeling choice by modifying expected utility theory. The present article pursues Tversky's original perspective, as well as the emphasis on sequential models by Luce, Selten, and Simon.

\section{Limits of the Model}

Our goal was to derive from empirical evidence a psychological process model that predicts choice behavior. Like all models, the priority heuristic is a simplification of real world phenomena. In our view, there are four major limitations: the existence of individual differences, low-stake ("peanuts") gambles, widely discrepant expected values, and problem representation.

Individual differences and low stakes. The priority heuristic embodies risk aversion for gains and risk seeking for losses. Even if the majority of people are risk averse in a particular situation, a minority will typically be risk seeking. Some of these risk lovers may focus on the maximum gain rather than on the minimum one as the first reason. Thus, the order of reasons is one potential source of individual differences; another one is the aspiration level that stops examination. We propose order and aspiration as two sources of individual differences. Moreover, risk seeking can also be produced by the properties of the choice problem itself. For instance, low stakes can evoke risk seeking for gains. Thus, low stakes can lead to the same reversal of the order of reasons as postulated before for individual differences.

Discrepant expected values. Another limiting condition for the application of the priority heuristic is widely discrepant expected values. The set of random gambles by Erev et al. (2002) revealed this limitation. For instance, gamble $A$ offers 88 with $p=$ .74 , otherwise nothing, and gamble $B$ offers 19 with $p=.86$, otherwise nothing. The expected values of these gambles are 65.1 and 16.3 , respectively. The priority heuristic predicts the choice of gamble $B$, whereas the majority of participants chose gamble $A$.

To investigate the relation between the ratio of expected values and the predictive power of the priority heuristic, we analyzed a set of 450 problems with a large variability in expected values (Mellers, Chang, Birnbaum, \& Ordóñez, 1992). In this set, all minimal outcomes are zero; thus the priority heuristic could not use its top-ranked reason. We also tested how well cumulative prospect theory, security-potential/aspiration theory, the transferof-attention-exchange model, and expected value theory predict the majority choices.

\footnotetext{
${ }^{7}$ For the first set of problems, there were 3 independent parameter sets for cumulative prospect theory, 1 for security-potential/aspiration theory, and 1 for the transfer-of-attention-exchange model, resulting in 5 comparisons. For the second set, these numbers were 2, 0 , and 1 ; for the third set, 2,1 , and 0 ; and for the fourth set, 2,1 , and 1 ; resulting in 15 comparisons.
} 
Figure 8 shows the proportion of correct predictions as a function of the ratio between expected values. ${ }^{8}$ As was suggested by our analysis of the Erev et al. (2002) set of problems, the priority heuristic's accuracy decreased as the ratio between expected values became large. For instance, in the fourth quartile, its performance was only slightly above $50 \%$. In the first quartile, however, the priority heuristic outperformed all other contestants by a minimum of 16 percentage points (security-potential/aspiration theory) and a maximum of 40 percentage points (transfer-ofattention-exchange model). In the second quartile, the priority heuristic still outperformed the other modifications of expected utility theory. These performed better than the priority heuristic when the ratio between expected values exceeded about two. It is interesting, however, that expected value theory performed virtually as well as the best-performing modification for larger ratios. Tallying (not shown in Figure 8) performed identically to securitypotential/aspiration theory in the first two quartiles and worse than any other model when the ratios between expected values were larger. Thus, the results suggest that when choices become difficult-because of similar expected values - a simple sequential heuristic performs best. When choices become easy-because of widely discrepant expected values-expected value theory predicts choices as well as or better than the parameterized models. Both the priority heuristic and expected value theory successfully predict behavior without transforming probabilities and outcomes.

Figure 8 suggests that people do not rely on the priority heuristic indiscriminately. How can we model when they rely on the heuristic and when they do not? One way would be to assume that people estimate the expected values, and if the ratio is smaller than

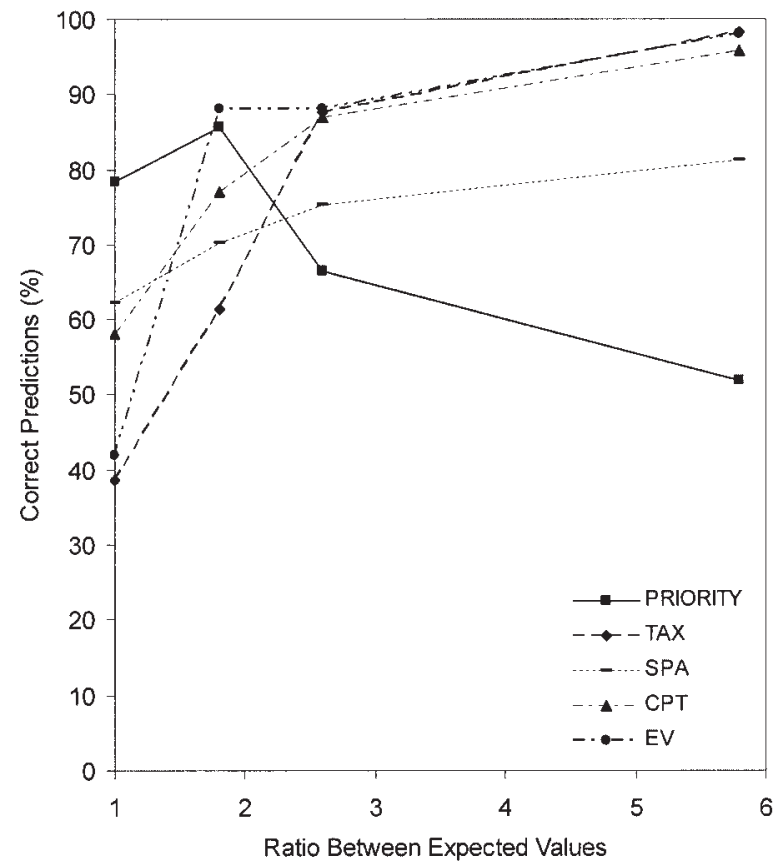

Figure 8. Correct predictions dependent on the ratio between expected values for the set of problems in Mellers et al. (1992). For parameter estimates, see Footnote 8. PRIORITY = priority heuristic; $\mathrm{TAX}=$ transfer-of-attention-exchange model; SPA $=$ security-potential/aspiration theory; $\mathrm{CPT}=$ cumulative prospect theory; $\mathrm{EV}=$ expected value theory. two, they turn to the priority heuristic. But calculating expected values is not the only method. Alternatively, people may first look at the three (four) reasons, and if no difference is markedly larger than the others, they apply the priority heuristic. Screening the reasons for a large difference is akin to what Tversky, Sattath, and Slovic (1988) called "looking for a decisive advantage" (p. 372).

Problem representation. A final potential limitation refers to the impact of different representations of the same decision problems on people's choices. For illustration, consider the following two problems reported by Birnbaum (2004):

A marble will be drawn from an urn, and the color of the marble drawn blindly and randomly will determine your prize. You can choose the urn from which the marble will be drawn. In each choice, which urn would you choose?

Urn $A: \quad 85$ red marbles to win $\$ 100$

10 white marbles to win $\$ 50$

5 blue marbles to win $\$ 50$

Urn $B: \quad 85$ black marbles to win $\$ 100$

10 yellow marbles to win $\$ 100$

5 purple marbles to win $\$ 7$

The same participants were also asked to choose between the following two urns:
Urn $A^{\prime}$ :
85 black marbles to win $\$ 100$
15 yellow marbles to win $\$ 50$
95 red marbles to win $\$ 100$
5 white marbles to win $\$ 7$

The Urn $A$ versus Urn $B$ problem is the same as the Urn $A^{\prime}$ versus Urn $B^{\prime}$ problem, except that the latter adds up the probabilities of the same outcomes (e.g., 10 white marbles to win $\$ 50$ and 5 blue marbles to win $\$ 50$ in Urn $A$ are combined to 15 yellow marbles to win $\$ 50$ in Urn $A^{\prime}$ ). According to Birnbaum (2004), $63 \%$ of his participants chose $B$ over $A$ and only $20 \%$ chose $B^{\prime}$ over $A^{\prime}$. His transfer-of-attention-exchange model predicts this and other new paradoxes of risky decision making (see also Loomes, Starmer, \& Sugden, 1991). The priority heuristic, in contrast, does not predict that such reversals will occur. The heuristic predicts that people prefer Urn $A$ and $A^{\prime}$, respectively (based on the minimum gains).

In evaluating the validity of models of risky choice, it is important to keep in mind that it is always possible to design problems that elicit choices that a given model-be it the expected utility theory, prospect theory, cumulative prospect theory, the transferof-attention-exchange model, or the priority heuristic-can and cannot explain. For this reason, we refrained from opportunistic sampling of problems. Instead, we tested the priority heuristic on

\footnotetext{
${ }^{8}$ For each problem, we calculated the ratio between the larger and the smaller expected value. We then divided the ratios into four quartiles and calculated the mean ratio for each quartile, which were 1.0, 1.8, 2.6, and 5.8. We used the same parameter estimates as in the four contests. For cumulative prospect theory, Figure 8 shows the mean performance across the three analyses using the parameter estimates from Erev et al. (2002), Lopes and Oden (1999), and Tversky and Kahneman (1992).
} 
a large set of existing problems that were initially designed to demonstrate the validity of several of its contestants.

\section{Process Models}

The priority heuristic is intended to model both choice and process: It not only predicts the outcome but also specifies the order of priority, a stopping rule, and a decision rule. As a consequence, it can be tested on two levels: choice and process. For instance, if a heuristic predicts choices well, it may still fail in describing the process, thus falsifying it as a process model. Models of choice that are not intended to capture the psychological processes (i.e., as-if models), however, can only be tested at the level of choice. In discussions with colleagues, we learned that there is debate about what counts as a process model for choice. For instance, whereas many people assume that cumulative prospect theory is mute about the decision process, some think the theory can be understood in terms of processes. Lopes (1995) explicitly clarified that the equations in theories such as securitypotential/aspiration theory are not meant to describe the process. She even showed that the outcomes of lexicographic processessimilar to those in the priority heuristic — can resemble those of modifications of subjective expected utility theories.

The priority heuristic can be seen as an explication of Rubinstein's (1988) similarity-based model (see also Leland, 1994; Mellers \& Biagini, 1994). The role of "similarity" in his model is here played by the aspiration level, and the priority rule imposes a fixed order on the reasons. Unlike the algebra in expected utility theory and its modifications, which assume weighting, summing, and exhaustive use of information, the priority heuristic assumes cognitive processes that are characterized by order, aspiration levels, and stopping rules. In Rubinstein's (2003) words, “we need to open the black box of decision making, and come up with some completely new and fresh modeling devices" (p. 1215). We believe that process models of heuristics are key to opening this black box.

\section{Predicting Choices: Which Strategies Are Closest?}

Which of the strategies make the same predictions and which make contradictory ones? Table 5 shows the percentage of identical predictions between each pair of strategies tested on the entire set of 260 problems. The strategy that is most similar to the priority heuristic in terms of prediction (but not in terms of process) is not a heuristic, but rather cumulative prospect theory using the parameters from the Erev et al. (2002) set of problems. The least similar strategy in terms of prediction is the equal weight heuristic, which, unlike the priority heuristic, ignores probabilities and simply adds the outcomes.

A second striking result concerns models with adjustable parameters. The degree of overlap in prediction is not so much driven by their conceptual similarity or dissimilarity as by whether they are fitted to the same set of problems. Consider first the cases in which the parameters of different models are derived from the same set of problems. The transfer-of-attention-exchange model (with parameter estimates from Tversky \& Kahneman, 1992) most closely resembles cumulative prospect theory when its parameters are estimated from the same set of problems (96\% identical predictions). Similarly, security-potential/aspiration theory (with parameter estimates from Lopes \& Oden, 1999) most closely resembles cumulative prospect theory when its parameters are estimated from the same problem set ( $91 \%$ identical predictions). Consider now the cases in which the parameters of the same model are derived from different sets of problems. There are three such cases

Table 5

Percentage of Same Predictions of Each Pair of Strategies

\begin{tabular}{|c|c|c|c|c|c|c|c|c|c|c|c|c|c|c|c|c|}
\hline Strategy & 1 & 2 & 3 & 4 & 5 & 6 & 7 & 8 & 9 & 10 & 11 & 12 & 13 & 14 & 15 & 16 \\
\hline 1. PRIORITY & - & & & & & & & & & & & & & & & \\
\hline 2. CPT; L\&O (1999) & 78 & - & & & & & & & & & & & & & & \\
\hline 3. CPT; Erev et al. (2002) & 89 & 92 & - & & & & & & & & & & & & & \\
\hline 4. CPT; T\&K (1992) & 68 & 80 & 60 & - & & & & & & & & & & & & \\
\hline 5. TAX & 65 & 77 & 57 & 96 & - & & & & & & & & & & & \\
\hline 6. SPA & 72 & 91 & 81 & 77 & 76 & - & & & & & & & & & & \\
\hline 7. MAXI & 38 & 40 & 26 & 57 & 58 & 44 & - & & & & & & & & & \\
\hline 8. MINI & 51 & 49 & 75 & 59 & 60 & 47 & 39 & - & & & & & & & & \\
\hline 9. TALL & 70 & 62 & 56 & 52 & 51 & 63 & 20 & 49 & - & & & & & & & \\
\hline 10. ML & 51 & 57 & 48 & 42 & 41 & 62 & 39 & 43 & 60 & - & & & & & & \\
\hline 11. LL & 64 & 62 & 48 & 38 & 36 & 66 & 36 & 32 & 81 & 67 & - & & & & & \\
\hline 12. BTA & 49 & 57 & 46 & 70 & 72 & 57 & 67 & 53 & 43 & 32 & 39 & - & & & & \\
\hline 13. EQUI & 43 & 43 & 51 & 64 & 70 & 41 & 77 & 59 & 19 & 22 & 19 & 74 & - & & & \\
\hline 14. EQW & 31 & 27 & 32 & 62 & 65 & 27 & 77 & 58 & 19 & 32 & 19 & 69 & 84 & - & & \\
\hline 15. PROB & 51 & 57 & 48 & 42 & 41 & 62 & 39 & 43 & 60 & 89 & 67 & 32 & 22 & 32 & - & \\
\hline 16. LEX & 50 & 53 & 44 & 44 & 42 & 60 & 44 & 44 & 51 & 89 & 62 & 33 & 28 & 37 & 89 & - \\
\hline
\end{tabular}

Note. The numbers specify the percentage of problems in which two strategies made the same predictions. For instance, the priority heuristic (PRIORITY) and minimax (MINI) made the same predictions in $51 \%$ of all problems and different predictions in $49 \%$ of them. Parameters for cumulative prospect theory (CPT) are estimated from Lopes and Oden (L\&O; 1999), Erev et al. (2002), and Tversky and Kahneman (T\&K; 1992), respectively. Italic numbers indicate the percentage of same predictions when the parameters of the same model are derived from different sets of problems. Bold numbers indicate the percentage of same predictions when the parameters of different models are derived from the same set of problems. TAX $=$ transfer-of-attention-exchange model; SPA = security-potential/aspiration theory; MAXI = maximax heuristic; TALL = tallying; ML = most-likely heuristic; $\mathrm{LL}=$ least-likely heuristic; $\mathrm{BTA}=$ better-than-average heuristic; $\mathrm{EQUI}=$ equiprobable heuristic; $\mathrm{EQW}=$ equal-weight heuristic; $\mathrm{PROB}=$ probable heuristic, $\mathrm{LEX}=$ lexicographic heuristic. For a description of the heuristics, see Table 3. 
for cumulative prospect theory, and the overlaps are $92 \%, 80 \%$, and $60 \%$. Thus, on average the overlap is higher (94\%) when the same problem set is used rather when the same model is used (77\%). This shows that the difference between problem sets has more impact than the difference between models does.

\section{Can the Priority Heuristic Predict Choices in Gambles Involving Gains and Losses?}

In the four contests, we have shown that the priority heuristic predicts choices by avoiding trade-offs between probability and outcomes; we have not investigated trade-offs between gains and losses. The priority heuristic can be generalized to handle gainloss trade-offs, without any change in its logic. This is illustrated by the following choice between two mixed gambles (Tversky \& Kahneman, 1992):
A:
$1 / 2$ probability to lose 50
$1 / 2$ probability to win 150
$B$ :
$1 / 2$ probability to lose 125
$1 / 2$ probability to win 225

The heuristic starts by comparing the minimum outcomes $(-50$ and -125). Because this difference exceeds the aspiration level of 20 (1/10 of the highest absolute gain or loss: 22.5 rounded to the next prominent number), examination is stopped, and no other reasons are examined. The priority heuristic selects gamble $A$, which is the majority choice (inferred from certainty equivalents as in Contest 3). Applied to all six choice problems with mixed gambles in the Tversky and Kahneman (1992) set of problems, the priority heuristic predicted the majority choice in each case. We cross-checked this result against the set of problems by Levy and Levy (2002), with six choices between mixed gambles with two, three, or four outcomes. Again, the priority heuristic predicted the majority choice in each case correctly. However, we did not test the proposed generalization of the priority heuristic against an extensive set of mixed gambles and thus cannot judge how appropriate this generalization is.

\section{Choice Proportions}

The priority heuristic predicts majority choices but not choice proportions. However, a rank order of choice proportion can be predicted with the additional assumption that the earlier the examination stops, the more extreme the choice proportions will be. That is, when examination is stopped after the first reason, the resulting choice proportions will be more unequal (e.g., 80/20) than when stopping occurs after the second reason (e.g., 70/30), and so on. To test this hypothesis, we analyzed two sets of problems (Kahneman \& Tversky, 1979; Lopes \& Oden, 1999) in which the priority heuristic predicted stopping after the first, second, or third reason (this was not the case for the Erev et al., 2002, choice problems, in which stopping after the first reason was not possible in almost all problems; the problems in the Tversky \& Kahneman, 1992, data set were derived from certainty equivalents and hence do not contain choice proportions). Thus, the hypothesis implies that the predicted choice proportions should be higher when fewer reasons are examined. The results show that if examination stopped after the first, second, and third reason, the respec- tive mean choice proportions are $.85(S D=.06 ; n=3), .83(S D=$ $.09 ; n=4)$, and $.72(S D=.09 ; n=7)$ in the Kahneman and Tversky (1979) set of problems. Similarly, in the Lopes and Oden (1999) set of problems, these values are $.75(S D=.10 ; n=30), .66(S D=.11$; $n=48)$, and $.54(S D=.03 ; n=12)$, which supports the heuristic's capacity to predict a rank order of choice proportions.

We suggest that the number of reasons examined offers one account for the process underlying the observed relationship between choice proportion and response time. Our analysis showed that when fewer reasons were examined, the choice proportions became more extreme and the response times decreased. This implies, everything else being equal, that more extreme choice proportions should be associated with faster response times. Some support for this implication is given in Mosteller and Nogee (1951) and in Busemeyer and Townsend (1993).

\section{Occam's Razor}

Models with smaller numbers of adjustable parameters, which embody Occam's razor, have a higher posterior probability in Bayesian model comparison (MacKay, 1995; Roberts \& Pashler, 2000). Consider an empirically obtained result that is consistent with two models. One of these predicts that behavior will be located in a small range of the outcome space, and the other predicts that behavior will be located in a wider range. The empirical result gives more support (a higher Bayesian posterior probability) to the one that bets on the smaller range. Consequently, if several models predict the same empirical phenomenon equally well, the simplest receives more support (Simon, 1977). We provided evidence that the priority heuristic (a) is simpler and more frugal than subjective expected utility and its modifications; (b) can predict choices equally well or better across four sets of gambles; (c) predicts intransitivities, which some modifications of expected utility theory have difficulty predicting; and (d) predicts process data such as response times.

Every model has parameters; the difference is whether they are free, adjustable within a range, or fixed. The parameters in modifications of expected utility theory are typically adjustable within a range, because of theoretical constraints. In contrast, most heuristics have fixed parameters. One can fix a parameter by (a) measuring it independently, (b) deriving it from previous research, or (c) deriving it theoretically. For modifications of expected utility theories, we used parameters measured on independent sets of problems. For the priority heuristic, we derived its order from previous research and obtained the 1/10 aspiration level from our cultural base-10 number system. These ways of fixing parameters can help to make more precise predictions, thus increasing the empirical support for a model.

\section{Fast and Frugal Heuristics: From Inferences to Preferences}

By means of the priority heuristic, we generalize the research program on fast and frugal heuristics (Gigerenzer et al., 1999) from inferences to preferences, thus linking it with another research program on cognitive heuristics, the adaptive decisionmaker program (Payne et al., 1993). This generalization is not trivial. In fact, according to a widespread intuition, preference judgments are not likely to be modeled in terms of noncompen- 
satory strategies such as the priority heuristic. The reason is that preferential choice often occurs in environments in which reasons-for example, prices of products and their quality-correlate negatively. Some researchers have argued that negative correlations between reasons cause people to experience conflict, leading them to make trade-offs, and trade-offs in turn are not conducive to the use of noncompensatory heuristics (e.g., Shanteau \& Thomas, 2000). The priority heuristic's success in predicting a large majority of the modal responses across 260 problems challenges this argument.

The study of fast and frugal heuristics for inferences has two goals. One is to derive descriptive models of cognitive heuristics that capture how real people actually make inferences. The second goal is prescriptive in nature: to determine in which environments a given heuristic is less accurate than, as accurate as, or even more accurate than informationally demanding and computationally expensive strategies. In the current analysis of a fast and frugal heuristic for preferences, we focused on the descriptive goal at the expense of the prescriptive one for the following reason: When analyzing preference judgments in prescriptive terms, one quickly enters muddy waters because, unlike in inference tasks, there is no external criterion of accuracy. Moreover, Thorngate (1980) and Payne et al. (1993) have already shown that in some environments, preference heuristics can be highly competitive-when measured, for instance, against the gold standard of a weighted additive model. Notwithstanding our focus on the descriptive accuracy of the priority heuristic, we showed that it performed well on two criteria that have also been used to evaluate the performance of fast and frugal inference strategies, namely, frugality and transparency.

Perhaps one of the most surprising outcomes of the contest between the priority heuristic, the neo-Bernouillian models (i.e., those assuming some type of weighing and summing of reasons), and previously proposed heuristics, respectively, is the dismal performance of the latter. Why does the priority heuristic so clearly outperform the other heuristics? The key difference is that the classic heuristics (with the exception of the lexicographic heuristic) always look at the same piece or several pieces of information. The priority heuristic, in contrast, relies on a flexible stopping rule. Like the classic heuristics, it is frugal, but unlike them, it is adapted to the specific properties of a problem and its frugality is hence not independent of the problem in question. The sequential nature of the priority heuristic is exactly the same as that assumed in heuristics such as Take The Best, Take The Last, and fast and frugal trees (Gigerenzer, 2004). These heuristics, equipped with flexible stopping rules, are in between the classic heuristics that always rely on the same reason(s) and the neo-Bernoulli models that use all reasons. We believe this new class of heuristics to be of great importance. Its heuristics combine some of the advantages of both classic trade-off models and heuristics, thus achieving great flexibility, which enables them to respond to the characteristics of individual problems.

\section{Conclusion}

We have shown that a person who uses the priority heuristic generates (a) the Allais paradox, (b) risk aversion for gains if probabilities are high, (c) risk seeking for gains if probabilities are low (e.g., lottery tickets), (d) risk aversion for losses if probabilities are low (e.g., buying insurance), (e) risk seeking for losses if probabilities are high, (f) the certainty effect, and (g) the possibility effect. Furthermore, the priority heuristic is capable of accounting for choices that conflict with (cumulative) prospect theory, such as systematic intransitivities that can cause preference reversals. We tested how well the heuristic predicts people's majority choices in four different types of gambles; three of these had been designed to test the power of prospect theory, cumulative prospect theory, and security-potential/aspiration theory, and the fourth was a set of random gambles. Nevertheless, despite this test in "hostile" environments, the priority heuristic predicted people's preference better than previously proposed heuristics and better than three modifications of expected utility theory. We also identified an important boundary of the priority heuristic. Specifically, the heuristic performed best when the ratio between expected values was about 2:1 or smaller. Finally, the heuristic specifies a process that leads to predictions about response time differences between choice problems, which we tested and confirmed.

We believe that the priority heuristic, which is based on the same building blocks as Take The Best, can serve as a new framework for models for a wide range of cognitive processes, such as attitude formation or expectancy-value theories of motivation. The heuristic provides an alternative to the assumption that cognitive processes always compute trade-offs in the sense of weighting and summing of information. We do not claim that people never make trade-offs in choices, judgments of facts, values, and morals; that would be as mistaken as assuming that they always do. Rather, the task ahead is to understand when people make trade-offs and when they do not.

\section{References}

Albers, W. (2001). Prominence theory as a tool to model boundedly rational decisions. In G. Gigerenzer \& R. Selten (Eds.), Bounded rationality: The adaptive toolbox (pp. 297-317). Cambridge. MA: MIT Press.

Allais, M. (1953). Le comportement de l'homme rationel devant le risque: Critique des postulats et axioms de l'école americaine [Rational man's behavior in face of risk: Critique of the American School's postulates and axioms]. Econometrica, 21, 503-546.

Allais, M. (1979). The so-called Allais paradox and rational decisions under uncertainty. In M. Allais \& O. Hagen (Eds.), Expected utility hypotheses and the Allais paradox (pp. 437-681). Dordrecht, the Netherlands: Reidel.

Arnauld, A., \& Nicole, P. (1996). Logic or the art of thinking. Cambridge, England: Cambridge University Press. (Original work published 1662)

Atkinson, J. W. (1957). Motivational determinants of risk-taking behavior. Psychological Review, 64, 359-372.

Banks, W. P., \& Coleman, M. J. (1981). Two subjective scales of number. Perception \& Psychophysics, 29, 95-105.

Becker, M. H. (1974). The health belief model and personal health behavior. Health Education Monographs, 2, 324-508.

Bell, D. E. (1982). Regret in decision making under uncertainty. Operations Research, 30, 961-981.

Bell, D. E. (1985). Disappointment in decision making under uncertainty. Operations Research, 33, 1-27.

Bernoulli, D. (1954). Exposition of a new theory on the measurement of risk. Econometrica, 22, 23-36. (Original work published 1738)

Birnbaum, M. (2004). Causes of Allais common consequence paradoxes: An experimental dissection. Journal of Mathematical Psychology, 48, 87-106.

Birnbaum, M., \& Chavez, A. (1997). Tests of theories of decision making: Violations of branch independence and distribution independence. $\mathrm{Or}$ ganizational Behavior and Human Decision Processes, 71, 161-194. 
Birnbaum, M., \& Navarrete, J. (1998). Testing descriptive utility theories: Violations of stochastic dominance and cumulative independence. Journal of Risk and Uncertainty, 17, 49-78.

Brandstätter, E. (2004). [Choice behavior in risky gambles]. Unpublished raw data.

Brandstätter, E., \& Kühberger, A. (2005). Outcome priority in risky choice. Manuscript submitted for publication.

Brandstätter, E., Kühberger, A., \& Schneider, F. (2002). A cognitiveemotional account of the shape of the probability weighting function. Journal of Behavioral Decision Making, 15, 79-100.

Bröder, A. (2000). Assessing the empirical validity of the "Take The Best" heuristic as a model of human probabilistic inference. Journal of Experimental Psychology: Learning, Memory, and Cognition, 26, 13321346.

Bröder, A. (2003). Decision making with the adaptive toolbox: Influence of environmental structure, intelligence, and working memory load. Journal of Experimental Psychology: Learning, Memory \& Cognition, 29, 611-625.

Bröder, A., \& Schiffer, S. (2003). "Take The Best" versus simultaneous feature matching: Probabilistic inferences from memory and effects of representation format. Journal of Experimental Psychology: General, 132, 277-293.

Busemeyer, J. R., \& Townsend, J. T. (1993). Decision field theory: A dynamic-cognitive approach to decision making in an uncertain environment. Psychological Review, 100, 432-459.

Camerer, C. (1995). Individual decision making. In J. H. Kagel \& A. E. Roth (Eds.), The handbook of experimental economics (pp. 587-703). Princeton, NJ: Princeton University Press.

Daston, L. J. (1988). Classical probability in the Enlightenment. Princeton, NJ: Princeton University Press.

Dawes, R. (1979). The robust beauty of improper linear models in decision making. American Psychologist, 23, 571-582.

de Finetti, B. (1979). A short confirmation of my standpoint. In M. Allais \& O. Hagen (Eds.), Expected utility hypotheses and the Allais paradox (p. 161). Dordrecht, the Netherlands: Reidel.

Deane, G. E. (1969). Cardiac activity during experimentally induced anxiety. Psychophysiology, 6, 17-30.

Dhami, M. K. (2003). Psychological models of professional decision making. Psychological Science, 14, 175-180.

Edwards, W. (1954). The theory of decision making. Psychological Bulletin, 51, 380-417.

Edwards, W. (1962). Subjective probabilities inferred from decisions. Psychological Review, 69, 109-135.

Edwards, W. (1968). Conservatism in human information processing. In B. Kleinmuntz (Ed.), Formal representation of human judgment (pp. 1752). New York: Wiley.

Ellsberg, D. (1961). Risk, ambiguity, and the Savage axioms. Quarterly Journal of Economics, 75, 643-699.

Erev, I., Roth, A. E., Slonim, R. L., \& Barron, G. (2002). Combining a theoretical prediction with experimental evidence to yield a new prediction: An experimental design with a random sample of tasks. Unpublished manuscript, Columbia University and Faculty of Industrial Engineering and Management, Techion, Haifa, Israel.

Fishbein, M., \& Ajzen, I. (1975). Belief, attitude, intention, and behavior: An introduction to theory and research. Reading, MA: Addison Wesley.

Fishburn, P. C. (1979). On the nature of expected utility. In M. Allais \& O. Hagen (Eds.), Expected utility hypotheses and the Allais paradox (pp. 243-257). Dordrecht, the Netherlands: Reidel.

Fishburn, P. C., \& Kochenberger, G. A. (1979). Two-piece von NeumannMorgenstern utility functions. Decision Sciences, 10, 503-518.

Gigerenzer, G. (2004). Fast and frugal heuristics: The tools of bounded rationality: In D. Koehler \& N. Harvey (Eds.), Handbook of judgment and decision making (pp. 62-88). Oxford, England: Blackwell.

Gigerenzer, G., \& Goldstein, D. G. (1996). Reasoning the fast and frugal way: Models of bounded rationality. Psychological Review, 103, 650669.

Gigerenzer, G., \& Kurz, E. (2001). Vicarious functioning reconsidered: A fast and frugal lens model. In K. R. Hammond \& T. R. Stewart (Eds.), The essential Brunswik: Beginnings, explications, applications (pp. 342-347). New York: Oxford University Press.

Gigerenzer, G., \& Murray, D. J. (1987). Cognition as intuitive statistics. Hillsdale, NJ: Erlbaum.

Gigerenzer, G., Swijtink, Z., Porter, T., Daston, L., Beatty, J., \& Krüger, L. (1989). The empire of chance: How probability changed science and everyday life. Cambridge, England: Cambridge University Press.

Gigerenzer, G., Todd, P. M., \& the ABC Research Group. (1999). Simple heuristics that make us smart. New York: Oxford University Press.

Hacking, I. (1975). The emergence of probability. New York: Cambridge University Press.

Heckhausen, H. (1991). Motivation and action (P. K. Leppmann, Trans.). Berlin: Springer-Verlag.

Hertwig, R., Barron, G., Weber, E. U., \& Erev, I. (2004). Decision from experience and the effect of rare events. Psychological Science, 15, $534-539$.

Hertwig, R., \& Ortmann, A. (2001). Experimental practices in economics. A methodological challenge for psychologists? Behavioral and Brain Sciences, 24, 383-451.

Hogarth, R. M., \& Karelaia, N. (2005). Ignoring information in binary choice with continuous variables: When is less "more"? Journal of Mathematical Psychology, 49, 115-124.

Hogarth, R. M., \& Reder, M. W. (Eds.). (1986). Rational choice. Chicago: University of Chicago Press.

Huber, O. (1982). Entscheiden als Problemlösen [Decision making as problem solving]. Bern, Switzerland: Hans Huber.

Kahneman, D. (2000). Preface. In D. Kahneman \& A. Tversky (Eds.), Choices, values, and frames (pp. ix-xvii). Cambridge, England: Cambridge University Press.

Kahneman, D., \& Tversky, A. (1979). Prospect theory: An analysis of decision under risk. Econometrica, 47, 263-291.

Kahneman, D., \& Tversky, A. (Eds.). (2000). Choices, values, and frames. Cambridge, England: Cambridge University Press.

Katsikopoulos, K. V., \& Martignon, L. (in press). Naive heuristics for paired comparisons: Some results on their relative accuracy. Journal of Mathematical Psychology.

Leland, J. W. (1994). Generalized similarity judgments: An alternative explanation for choice anomalies. Journal of Risk and Uncertainty, 9, 151-172.

Levy, M., \& Levy, H. (2002). Prospect theory: Much ado about nothing? Management Science, 48, 1334-1349.

Loewenstein, G. F., Weber, E. U., Hsee, C. K., \& Welch, N. (2001). Risk as feeling. Psychological Bulletin, 127, 267-286.

Loomes, G., Starmer, C., \& Sugden, R. (1991). Observing violations of transitivity by experimental methods. Econometrica, 59, 425-439.

Loomes, G., \& Sugden, R. (1982). Regret theory: An alternative theory of rational choice under uncertainty. The Economic Journal, 92, 805-824.

Loomes, G., \& Sugden, R. (1986). Disappointment and dynamic consistency in choice under uncertainty. Review of Economic Studies, 53, 271-282.

Lopes, L. L. (1987). Between hope and fear: The psychology of risk. Advances in Experimental Social Psychology, 20, 255-295.

Lopes, L. L. (1995). Algebra and the process in the modeling of risky choice. The Psychology of Learning and Motivation, 32, 177-220.

Lopes, L. L., \& Oden, G. C. (1999). The role of aspiration level in risky choice: A comparison of cumulative prospect theory and SP/A theory. Journal of Mathematical Psychology, 43, 286-313.

Luce, R. D. (1956). Semiorders and a theory of utility discrimination. Econometrica, 24, 178-191.

MacCrimmon, K. R. (1968). Descriptive and normative implications of the 
decision-theory postulate. In K. H. Borch \& J. Mossin (Eds.), Risk and uncertainty (pp. 3-23). New York: St. Martin's Press.

MacKay, D. J. C. (1995). Probable networks and plausible predictions-A review of practical Bayesian methods for supervised neural networks. Network: Computation in Neural Systems, 6, 469-505.

Markowitz, H. (1952). The utility of wealth. Journal of Political Economy, 60, 151-158.

Martignon, L., \& Hoffrage, U. (2002). Fast, frugal, and fit: Simple heuristics for paired comparison. Theory and Decision, 52, 29-71.

Mellers, B. A. (2000). Choice and the relative pleasure of consequences. Psychological Bulletin, 126, 910-924.

Mellers, B. A., \& Biagini, K. (1994). Similarity and choice. Psychological Review, 101, 505-518.

Mellers, B. A., Chang, S., Birnbaum, M. H., \& Ordóñez, L. D. (1992). Preferences, prices, and ratings in risky decision making. Journal of Experimental Psychology: Human Perception and Performance, 18, 347-361.

Menger, C. (1990). Grundsätze der Volkswirtschaftslehre [Principles of economics]. Dusseldorf, Germany: Wirtschaft und Finanzen. (Original work published 1871)

Mosteller, F., \& Nogee, P. (1951). An experimental measurement of utility. The Journal of Political Economy, 59, 371-404.

Newell, B. R., Weston, N. J., \& Shanks, D. R. (2003). Empirical tests of a fast-and-frugal heuristic: Not everyone "takes-the-best." Organizational Behavior and Human Decision Processes, 91, 82-96.

Payne, J. W., Bettman, J. R., \& Johnson, E. J. (1993). The adaptive decision maker. Cambridge, England: Cambridge University Press.

Payne, J. W., Bettman, J. R., \& Luce, M. F. (1996). When time is money: Decision behavior under opportunity-cost time pressure. Organizational Behavior and Human Decision Processes, 66, 131-152.

Payne, J. W., \& Braunstein, M. L. (1971). Preferences among gambles with equal underlying distributions. Journal of Experimental Psychology, 87, $13-18$.

Preston, M. G., \& Baratta, P. (1948). An experimental study of the auction value of an uncertain outcome. American Journal of Psychology, 61, 183-193.

Quiggin, J. (1982). A theory of anticipated utility. Journal of Economic Behavior and Organization, 3, 323-343.

Rachlinski, J. J. (1996). Gains, losses, and the psychology of litigation. Southern California Law Review, 70, 113-119.

Rieskamp, J., \& Hoffrage, U. (1999). When do people use simple heuristics and how can we tell? In G. Gigerenzer, P. M. Todd, \& the ABC Group, Simple heuristics that make us smart (pp. 141-167). New York: Oxford University Press.

Roberts, S., \& Pashler, H. (2000). How pervasive is a good fit? A comment on theory testing. Psychological Review, 107, 358-367.

Rotter, J. B. (1954). Social learning and clinical psychology. Englewood Cliffs, NJ: Prentice Hall.

Rubinstein, A. (1988). Similarity and decision-making under risk (Is there a utility theory resolution to the Allais-paradox?). Journal of Economic Theory, 46, 145-153.
Rubinstein, A. (2003). Economics and psychology? The case of hyperbolic discounting. International Economic Review, 44, 1207-1216.

Savage, L. J. (1954). The foundations of statistics (2nd ed.). New York: Dover.

Schkade, D. A., \& Johnson, E. J. (1989). Cognitive processes in preference reversals. Organizational Behavior and Human Decision Processes, 44, 203-231.

Selten, R. (2001). What is bounded rationality? In G. Gigerenzer \& R. Selten (Eds.), Bounded rationality: The adaptive toolbox (pp. 13-36). Cambridge, MA: MIT Press.

Shanteau, J., \& Thomas, R. P. (2000). Fast and frugal heuristics: What about unfriendly environments? Behavioral and Brain Sciences, 23, $762-763$.

Simon, H. A. (1957). Models of man. New York: Wiley

Simon, H. A. (1977). On judging the plausibility of theories. In H. A. Simon (Ed.), Models of discovery (pp. 25-45). Dordrecht, the Netherlands: Reidel.

Simon, H. A. (1983). Reason in human affairs. Stanford, CA: Stanford University Press.

Slovic, P., Griffin, D., \& Tversky, A. (1990). Compatibility effects in judgment and choice. In R. M. Hogarth (Ed.), Insights in decision making. A tribute to Hillel J. Einhorn. Chicago: University of Chicago Press.

Slovic, P., \& Lichtenstein, S. (1968). Relative importance of probabilities and payoffs in risk taking. Journal of Experimental Psychology Monographs, 78, 1-18.

Sunstein, C. R. (2003). Terrorism and probability neglect. Journal of Risk and Uncertainty, 26, 121-136.

Thorngate, W. (1980). Efficient decision heuristics. Behavioral Science, $25,219-225$.

Tversky, A. (1969). Intransitivity of preference. Psychological Review, 76, $31-48$.

Tversky, A. (1972). Elimination by aspects: A theory of choice. Psychological Review, 79, 281-299.

Tversky, A., \& Fox, C. R. (1995). Weighing risk and uncertainty. Psychological Review, 102, 269-283.

Tversky, A., \& Kahneman, D. (1992). Advances in prospect theory: Cumulative representation of uncertainty. Journal of Risk and Uncertainty, 5, 297-323.

Tversky, A., Sattath, S., \& Slovic, P. (1988). Contingent weighting in judgment and choice. Psychological Review, 95, 371-384.

von Neumann, J., \& Morgenstern, O. (1947). Theory of games and economic behavior. Princeton, NJ: Princeton University Press.

Vroom, V. H. (1964). Work and motivation. New York: Wiley.

Wigfield, A., \& Eccles, J. S. (1992). The development of achievement task values: A theoretical analysis. Developmental Review, 12, 265-310.

Williams, A. C. (1966). Attitudes toward speculative risks as an indicator of attitudes toward pure risks. Journal of Risk and Insurance, 33, $577-586$.

Yaari, M. E. (1987). The dual theory of choice under risk. Econometrica, 55, 95-115. 


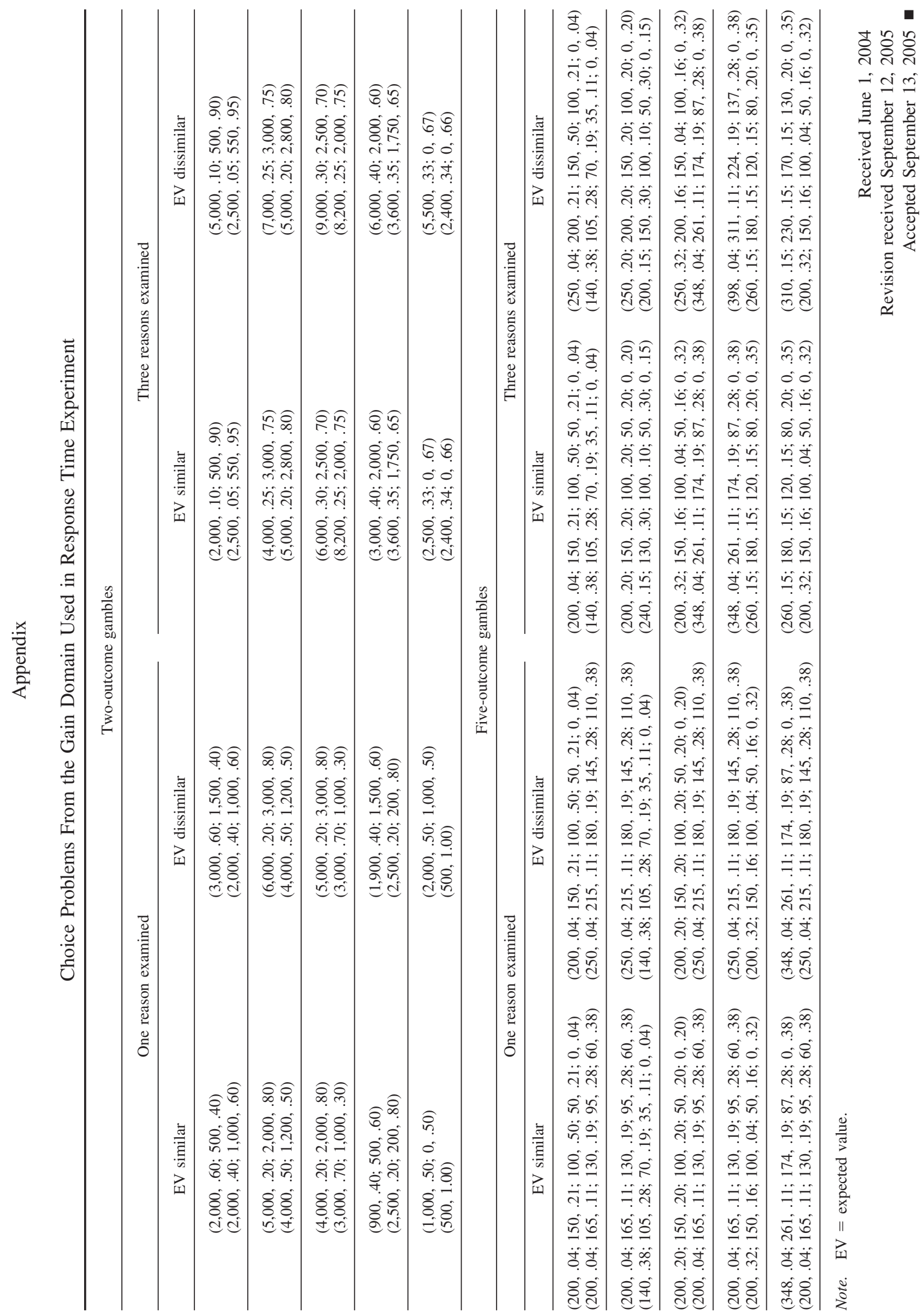

